\title{
«Éclairer l'action»? La démographie selon Alfred Sauvy au fil des rééditions de La Population
}

\author{
JACQUES VÉRON ${ }^{1}$
}

\begin{abstract}
Résumé
En 1944, l'économiste et démographe français Alfred Sauvy publie La population, un petit ouvrage d'initiation aux questions démographiques qui rencontre un très vif succès puisqu'il fait l'objet de quinze éditions, la dernière datant de 1992. Le corpus que constitue l'ensemble de ces éditions, complété par les autres ouvrages de cet auteur, permet de préciser la pensée démographique de Sauvy et son évolution sur près de cinquante années. Désireux d'éclairer l'action, il entendait montrer qu'un bon usage de la démographie permettait d'éviter nombre d'erreurs communes. La prévision démographique ou plus exactement l'élaboration de perspectives de population, que Sauvy considérait être au cœur du travail du démographe, permet de dessiner l'avenir le plus vraisemblable, compte tenu des évolutions en cours. Si celui-ci ne semble pas satisfaisant eu égard au bien-être collectif, des actions correctrices sont alors à envisager : des politiques de population, visant la natalité, la mortalité et/ou les migrations doivent dans ce cas être mises en place. Une certaine proximité du pouvoir, un natalisme revendiqué et des opinions tranchées sur les questions de population font de la démographie telle que l'envisage Sauvy une science politique plus que sociale, d'autant plus qu'il ne cesse de militer pour des interventions publiques.
\end{abstract}

\section{Mots-clés}

Démographie, problème de population, prévision, croissance, équilibre, politique de population, natalité, vieillissement, migration.

\section{Abstract}

In 1944, the French economist and demographer Alfred Sauvy published La Population, a highly successful short introduction to demographic questions which was reprinted no fewer than 15 times up until 1992. The corpus of these 15 editions, along with Alfred Sauvy's other books, give a detailed picture of his demographic thinking and its evolution over almost 50 years. Seeking to give meaning to action,

1. INED, 133 boulevard Davout, 75980 Paris cedex 20, France. L'auteur tient à remercier les referees pour leurs précieux commentaires. 
Sauvy aimed to show that numerous common errors can be avoided through the intelligent use of demography. For Sauvy, demographic forecasting or, more specifically, population projections, were central to the demographer's work, providing a means to predict the most likely future scenario while taking account of ongoing trends. If this scenario appears to compromise collective well-being, appropriate corrective action can be taken: population policies aiming to modify birth rates, death rates and/or migration must be set in place. With his proximity to the corridors of power, his clearly pro-natalist stance and his strong opinions on population issues, Sauvy - a long-standing campaigner for public intervention - saw demography as a political rather than a social science.

\section{Key words}

Demography, population issue, population projection, growth equilibrium, population policy, natality, ageing, migration.

En 1944, Alfred Sauvy publie aux Presses universitaires de France (Puf), dans la collection «Que sais-je ?» fondée trois ans plus tôt par Paul Angoulvent, La population, ouvrage d'introduction aux questions démographiques. C'est le 148ème numéro d'une collection qui compte aujourd'hui quelque 3800 titres.

Entre la date de la première édition et 1992, La population fait l'objet de 15 éditions. Certaines sont de simples rééditions tandis que d'autres sont des mises à jour ou des révisions. Destiné à un très large public, ce petit ouvrage se veut avant tout pédagogique : Sauvy y poursuit un objectif d'initiation aux questions de population. Mais la présentation faite de celles-ci, comme les exemples choisis et les comparaisons effectuées, témoignent aussi de la conception que Sauvy se faisait de la démographie. L'évolution du corps du texte et des références au fil des rééditions permet également de mettre en évidence des changements de contextes démographiques, de pointer l'apparition de nouveaux "problèmes», de voir dans quelle mesure il est fait état de nouveaux développements scientifiques et de suivre l'évolution de la pensée démographique de l'auteur.

C'est une double lecture de La population qui est proposée ici. Comment Sauvy présentait-il la démographie à un large public et quels nouveaux apports jugeait-il bon d'intégrer au fil du temps dans cet ouvrage pour rendre compte des progrès de la discipline ou faire état de nouvelles questions en débat, sachant que le format contraint de l'ouvrage (128 pages) obligeait à effectuer de réels choix rédactionnels ? Et comment la pensée démographique de Sauvy a-t-elle évolué sur près de 50 ans ? Audelà d'une initiation aux questions de population qui se voudrait «neu- 
tre», quels messages cherchait-il à faire passer auprès de ses lecteurs ? Dans l'esprit et sous la plume de Sauvy, quelle part de cette discipline mettait en jeu un système de valeurs ou, façon un peu différente de poser la question, dans quelle mesure sa conception de la démographie faisait-elle d'elle plutôt une science sociale ou plutôt une science politique, pour reprendre le distinguo de Dennis Hodgson (1983) ?

«Éclairer l'action». Ce fut un leitmotiv de Sauvy tout au long de son existence. En fait une véritable obsession, comme il l'avoue lui-même dans La vie en plus (Sauvy, 1981). Dans ce livre de souvenirs, il revient à plusieurs reprises sur cette vocation qui était la sienne, écrivant ainsi : "[é]clairer l'action sans la commander, telle a été ma devise, ma raison d'être». Si l'on en croit Sauvy, il fut un observateur attentif, un analyste scrupuleux, précisant à chaque fois qu'il le pouvait ce que la démographie permettait vraiment de «dire», à la façon de Nathan Keyfitz dans son article «Population Appearance and Demographic Reality» (Keyfitz, 1980) et de nous-même dans Arithmétique de l'Homme (Véron, 1993). En fait, Sauvy fut un économiste et démographe «engagé», ne cessant non seulement de dénoncer ce qu'il jugeait être des «erreurs» ou des manques de clairvoyance mais militant également pour que certaines décisions soient prises ou certaines actions entreprises. La connaissance n'allait pour lui pas sans l'action.

La lecture proposée ici des différentes éditions de La population se fera en suivant dans le même temps le parcours professionnel et intellectuel de Sauvy, de manière à mettre en quelque sorte en perspective ce petit ouvrage d'introduction aux questions de population.

\section{Une proximité du pouvoir}

Né le 31 octobre 1898 dans les Pyrénées orientales, reçu à l'École polytechnique en 1920, Sauvy entre à la Statistique Générale de la France (SGF) en $1922^{2}$. Il y élabore les premières perspectives démographiques de la France, avant d'assurer, à partir de 1929, le suivi de la conjoncture économique. Dans les années 1930, il devient proche d'Adolphe Landry, figure de la IIIème République, député radical socialiste de Corse, ministre de la marine en 1920, brièvement ministre de l'instruction publique

2. Pour les aspects concernant la vie d'Alfred Sauvy, le lecteur pourra utilement se référer aux «souvenirs» de ce dernier (Sauvy, 1981) ainsi qu'à la biographie de Michel-Louis Lévy, Alfred Sauvy compagnon du siècle (Lévy, 1990). 
en 1924 puis ministre du travail en 1932 . À ce poste, Landry s'occupe de l'application de la loi sur les assurances sociales. Grand défenseur de la cause des familles, il fut membre du Haut Comité de la Population créé en 1939. Un rapport de ce Haut Comité déboucha cette année-là sur le Code de la famille, véritable politique de population, prévoyant en particulier une aide aux familles. Sauvy restera très proche de Landry jusqu'à la mort de ce dernier (1956) dont il publiera une nécrologie particulièrement élogieuse dans un numéro de la revue Population (Sauvy, 1956). Rappelons qu'Adolphe Landry est aussi connu pour la publication de La Révolution démographique (Landry, 1934), amorce de ce qui deviendra la théorie de la transition démographique.

C'est par Landry que Sauvy rencontre Paul Reynaud, ministre des finances et très éphémère président du Conseil (équivalent de premier ministre) en 1940. Sauvy devient son conseiller économique. En 1939, il est nommé membre du Haut comité de la population. Dans ces années mais aussi par la suite, Sauvy croise de très nombreux hommes politiques, de Léon Blum (président du conseil en 1936, 1938 et 1946) à Edouard Daladier (président du conseil de 1938 à 1940), de Pierre Mendès-France (président du conseil en 1954-1955) aux présidents de la IVème puis de la Vème République 3 .

À la veille de la Seconde Guerre mondiale, Sauvy prend la direction de l'Institut de conjoncture, créé à Paris par un décret de 1938 et dont une des tâches est de produire des statistiques industrielles. Sauvy restera à la tête de cet institut jusqu'en 19454. En 1945, il est nommé secrétaire général à la Famille et à la Population par le Général de Gaulle. Il participe aux séances du Haut-Comité de la population recréé en $1945^{5}$ et que préside de Gaulle lui-même. Il devient peu après directeur de l'Institut national d'études démographiques (Ined) qui vient d'être créé par une ordonnance du 24 octobre 1945. Cet institut avait été imaginé par Robert Debré6 comme une transformation de la Fondation française pour

3. De Gaulle réagit par courrier à un envoi qui lui a été fait de La montée des jeunes ; il entame sa lettre par un «Mon cher Sauvy» et déclare être en accord avec celuici que «'l'accueil' des jeunes, c'est pour ainsi dire, tout l'essentiel du problème économique et social français» (Sauvy, 1972).

4. Dans ses mémoires, Alfred Sauvy se montre discret sur cette période de sa vie (Sauvy, 1981).

5. Par un décret du mois d'avril, le Haut comité de la population est devenu le Haut Comité consultatif de la population et de la famille.

6. Médecin, pédiatre de renom, le professeur Robert Debré fut à l'origine de la réforme conduisant en France à la création des centres hospitalo-universitaires (CHU). Son fils, Michel Debré, fut premier ministre du Général de Gaulle de 1959 à 1962. 
l'étude des problèmes humains dite «Fondation Carrel»7. L'Ined, qui reprend une partie du personnel de celle-ci, a pour vocation de mener des travaux ayant «une assise scientifique indiscutable» et «conduits avec une parfaite objectivité». Le champ d'investigation est réduit puisqu'il s'agit de traiter des seuls problèmes de population mais en conservant cependant une approche multidisciplinaire à laquelle Sauvy tient beaucoup. Dans une sorte d'introduction en tête du premier volume de la revue Population créée en 1946, Sauvy affiche une conception large d'une discipline qui se doit, selon lui, d'être utile :

\begin{abstract}
«On donne souvent à la démographie un sens restreint, en lui demandant seulement l'étude descriptive des populations, du simple point de vue quantitatif. L'Institut national d'études démographiques doit étendre plus loin son champ d'activité. Chargé d'éclairer l'action, en matière de politique de population, il doit aborder les phénomènes qualitatifs et attacher aux facteurs de causalité une attention particulière» (Sauvy, 1946).
\end{abstract}

Sauvy restera pendant 17 ans à la tête de cet institut, assurant la rédaction en chef de la revue Population publiée par l'Ined de la date de sa création jusqu'en 1976. Conservant un bureau à l'Ined, il restera actif presque jusqu'à sa mort le 30 octobre 1990, la veille de son 92ème anniversaire.

Personnage central de la démographie française du 20ème siècle ${ }^{8}, \mathrm{Al}-$ fred Sauvy l'est encore à d'autres titres. Il représente à partir de 1947 et pendant plus de 25 ans la France à la Commission de la Population des Nations Unies ( $c f$. infra, note 18). En 1947, il devient membre du Conseil économique transformé par la suite en Conseil économique et social ; il y siègera 27 ans. Il donne un cours intitulé «Population et économie» à l'école d'application de l'Institut national de la statistique et des études économiques (INSEE) nouvellement créé. Il enseigne aussi à l'Institut d'Études Politiques de Paris, à l'École nationale d'administration, à l'École polytechnique et à l'Université catholique de Louvain ${ }^{9}$. Pendant quelques années, il dirige l'Institut de démographie de l'Université de Paris

7. Alexis Carrel, chirurgien et biologiste récompensé par le prix Nobel de physiologie ou médecine en 1912 est l'auteur en 1935 de L'Homme, cet inconnu, ouvrage qui connut un retentissement mondial. A la Libération il fut une des cibles du Gouvernement provisoire de la République française pour sa proximité avec le régime de Vichy.

8. S'il a joué un rôle important dans le monde de la démographie, Sauvy avouait se sentir plus économiste ou statisticien-économiste que démographe (Sauvy, 1981; Lévy, 1990).

9. Le rôle joué par Sauvy dans la diffusion des connaissances démographiques et en particulier son soutien à l'enseignement de cette discipline, en France et à l'étranger, a été bien précisé par Alfred Dittgen (1992). 
(IDUP) ; il continue ensuite d'y enseigner l'économie de la population jusqu'à un âge avancé.

TABleaU 1 Titres de présentation d'Alfred Sauvy lors des différentes éditions de La population

\begin{tabular}{|c|c|c|c|}
\hline Année & Éditions & \multicolumn{2}{|c|}{ Titres de présentation } \\
\hline 1944 & 1ère & $\begin{array}{l}\text { Directeur de l'Institut de con- } \\
\text { joncture }\end{array}$ & $\begin{array}{l}\text { Ancien membre du Haut-Comité de } \\
\text { la Population }\end{array}$ \\
\hline 1948 & 2ème & $\begin{array}{l}\text { Directeur de l'Institut national } \\
\text { d'Études Démographiques }\end{array}$ & $\begin{array}{l}\text { Membre de la Commission de la } \\
\text { Population aux Nations-Unies }\end{array}$ \\
\hline 1953 & 3ème & Id. & $\begin{array}{l}\text { Ancien président de la Commission } \\
\text { de la Population aux Nations-Unies }\end{array}$ \\
\hline 1957 & 4ème & Id. & Id. \\
\hline 1959 & 5ème & Id. & Professeur au Collège de France \\
\hline 1961 & 6ème & Id. & Id. \\
\hline 1963 & 7ème & $\begin{array}{l}\text { Président honoraire de l'Union } \\
\text { Internationale pour l'Étude } \\
\text { Scientifique de la Population }\end{array}$ & Id. \\
\hline 1966 & $\begin{array}{l}\text { 8ème revue* } \\
\text { et mise à jour }\end{array}$ & Id. & Id. \\
\hline 1968 & 9ème mise à jour & Id. & Id. \\
\hline 1970 & 10ème, mise à jour & Id. & $\begin{array}{l}\text { Professeur honoraire au Collège de } \\
\text { France }\end{array}$ \\
\hline 1973 & 11ème, mise à jour & Id. & Id. \\
\hline 1975 & 12ème, mise à jour & Id. & Id. \\
\hline 1979 & 13ème & Id. & Id. \\
\hline 1984 & 14ème, mise à jour & Id. & Id. \\
\hline 1992 & 15 ème** & Id. & Id. \\
\hline
\end{tabular}

* La révision indiquée n'a pas de signification particulière, le contenu du volume étant susceptible de varier d'une édition à l'autre, quelles que soient celles-ci. ** II s'agit d'une édition posthume.

De 1961 à 1963, il préside l'Union internationale pour l'étude scientifique de la population (UIESP), après avoir été membre du bureau de l'Union de 1949 à 1953. Faisant le constat d'une faible représentation de scientifiques de pays de l'Est parmi les membres de l'UIESP, Sauvy entend rendre l'association plus internationale qu'elle n'est ${ }^{10}$. Il est fait docteur honoris causa de plusieurs universités étrangères. Élu professeur au Collège de France en 1959, il occupe pendant dix ans une chaire

10. Lors de l'Assemblée générale de l'Union qui se tient à Ottawa le 21 août 1963, Sauvy s'exprime ainsi: «les sujets de souci ou de non satisfaction ne manquent pas. Parmi eux, je dois placer au premier rang le regret de ne pas voir nos rangs comprendre un nombre plus élevé de savants des pays socialistes et notamment de l'Union soviétique. Nous ne ménagerons nos efforts pour réparer cette disproportion ; notre association ne méritera pleinement son titre d'internationale ou de mondiale que lorsque tous les pays seront convenablement représentés». («Le Démographe», Bulletin de l'Union internationale pour l'étude scientifique de la population, 12, octobre 1964). 
qu'il intitule «Démographie sociale» puis «La vie des populations». Par ailleurs, Sauvy collabore à plusieurs magazines tels que L'Express ou L'expansion et il tient de 1964 à 1986 une rubrique «Notes de lecture» dans le supplément économique hebdomadaire du quotidien Le Monde.

Les différentes éditions de La population permettent de suivre la façon dont Sauvy a souhaité se présenter sur un plan professionnel au cours de ces quelque cinquante années (Tableau 1). Ces présentations sont dans l'ensemble les mêmes que dans les autres ouvrages publiés aux dates correspondantes si ce n'est que, lorsqu'il y enseignait, Sauvy ne se présentait que comme professeur au Collège de France (même quand il était aussi directeur de l'Ined). Par la suite, quand un titre figurait sous son nom, ce qui n'était pas toujours le cas, c'était professeur honoraire au Collège de France.

\section{Sauvy : nataliste ou populationniste ?}

Avant de nous livrer à l'exégèse des différentes éditions de La population, il ne paraît pas inutile de rappeler que Sauvy est resté tout au long de son existence très préoccupé par la natalité qu'il jugeait trop faible. Dans Des Français pour la France, livre écrit au lendemain de la Seconde Guerre mondiale, Debré et Sauvy affichent on ne peut plus clairement leur natalisme en écrivant en particulier : «[p]our être libre, pour regarder son avenir en face, la France doit être riche d'enfants. Il n'y aura de vie heureuse pour les Français que si la France est une nombreuse famille» (Debré, Sauvy, 1946). Paul-André Rosental (2003) présente dans son Intelligence démographique, la nébuleuse du natalisme en France, avec comme personnages clés Landry, Debré, ... et Sauvy.

Dans la biographie qu'il lui a consacrée, Michel-Louis Lévy (1990) considère que Sauvy était davantage «populationniste» que strictement «nataliste», puisqu'il militait pour un accroissement de la population. $\mathrm{Ce}$ dernier devait être en priorité obtenu par une natalité plus élevée que celle observée, mais le recours à des migrations internationales était aussi recommandé :

«L'accroissement de la natalité ne peut donc suffire pour que nous atteignons notre but [l'accroissement de la population française], pas plus du reste que la baisse de la mortalité. Il faut donc recourir aussi à l'immigration» (Debré, Sauvy, 1946).

Toutefois, pour Sauvy «il faut aussi 'doser' les nationalités, les professions». S'il considère l'immigration indispensable, il évoque à plusieurs 
reprises un «problème d'assimilation» et se montre finalement partisan, dès cette fin des années 1940, d'une «immigration dirigée».

Venons-en maintenant à ce «Que-Sais-Je ?» dont la longévité a été remarquable afin d'analyser les évolutions de son contenu sur un demisiècle.

\section{La population : 15 éditions sur près de 50 ans}

De format réduit, les «Que sais-je ?» ont pour vocation collective de constituer une encyclopédie. C'était bien l'intention initiale de Paul Angoulvent lorsqu'il créa en 1941 cette collection. Les volumes sont rédigés par des spécialistes des différentes disciplines. Parmi les auteurs de «Que sais-je ?» figurent des personnalités reconnues comme Émile Borel, Raymond Boudon, Maurice Duverger, Jean Piaget, Jean Fourastié, François Perroux, Pierre Georges, Jacques Ellul, Emmanuel Leroy-Ladurie, etc. Le succès de certains ouvrages a longtemps fait de cette collection des Presses universitaires de France une collection de référence. Or, parmi les 26 titres qui avaient dépassé 100000 exemplaires vendus en 1975, figure La population d'Alfred Sauvy (Feller, 1975) ${ }^{11}$.

La préoccupation que les questions démographiques soient accessibles à un large public est constante chez Sauvy, désireux ainsi que la revue Population «ne s'adresse pas seulement aux démographes spécialisés, mais encore à tous ceux qui se préoccupent des grands problèmes d'intérêt national» (Sauvy, 1946).

Il est à noter que si le titre de l'ouvrage reste bien le même de 1944 à 1992, le sous-titre titre change à deux reprises. En 1944, le titre complet de l'ouvrage est La population. Ses lois, ses équilibres ; il devient La population. Ses mouvements, ses lois en 1957 et La population. Sa mesure, ses mouvements en 1961. Ce dernier sous-titre sera par la suite conservé jusqu'à la dernière réédition. L'ouvrage conserve la même structure sur toute la période, même si les intitulés évoluent quelque peu : il se compose de trois grandes parties présentant les aspects formels de la démographie, puis les faits, pour finir par les théories (Tableau 2). Quant à l'introduction, relativement longue puisqu'elle occupait cinq pages dans l'édition de 1944, elle est réduite à trois pages en 1957 (4ème édition)

11. La population fait l'objet d'une traduction en espagnol et en japonais. Dans la suite du texte, l'ouvrage La Population est cité par l'abréviation LP suivi de l'année de publication. 
grâce à un certain nombre de suppressions d'ordre historique en particulier, puis à 2 pages en 1961 (6ème édition) grâce à un changement de corps du texte, le contenu restant le même.

TABleau 2 Comparaison entre les sommaires des éditions de 1944, de 1968 et 1992 (entre parenthèses figurent les nombres de pages consacrées à chaque sujet)

\begin{tabular}{|c|c|c|}
\hline 1944 (1ère édition) & 1968 (9ème édition) & 1992 (15ème édition) \\
\hline Introduction (5) & Introduction (2) & Introduction (2) \\
\hline Première partie & Première partie & Première partie \\
\hline Méthodes démographiques (2) & Méthodes et instruments (2) & Méthodes et instruments (2) \\
\hline $\begin{array}{l}\text { Chap. I : Données de base et } \\
\text { méthodes de mesure (10) }\end{array}$ & $\begin{array}{l}\text { Chap. I : Rassemblement } \\
\text { des données de base }(10)\end{array}$ & $\begin{array}{l}\text { Chap. I : Rassemblement des } \\
\text { données de base (10) }\end{array}$ \\
\hline $\begin{array}{l}\text { Chap. II : État d'une Population } \\
\text { (9) }\end{array}$ & $\begin{array}{l}\text { Chap. II : État de la population } \\
\text { (6) }\end{array}$ & $\begin{array}{l}\text { Chap. II : État de la Population } \\
\text { (7) }\end{array}$ \\
\hline Chap. III : Mortalité (7) & Chap. III : Mortalité (7) & Chap. III : Mortalité (7) \\
\hline Chap. IV : Natalité (8) & Chap. IV : Natalité, fécondité (8) & Chap. IV : Natalité, fécondité (8) \\
\hline $\begin{array}{l}\text { Chap. V : Mouvement général } \\
\text { d'une population (7) }\end{array}$ & $\begin{array}{l}\text { Chap. V : Mouvement général } \\
\text { d'une population (6) }\end{array}$ & $\begin{array}{l}\text { Chap. V : Mouvement général } \\
\text { d'une population (6) }\end{array}$ \\
\hline $\begin{array}{l}\text { Chap VI : Prévisions démogra- } \\
\text { phiques (4) }\end{array}$ & $\begin{array}{l}\text { Chap. VI: Projections, perspec- } \\
\text { tives démographiques }(6)\end{array}$ & $\begin{array}{l}\text { Chap. VI : Projections, perspec- } \\
\text { tives démographiques (6) }\end{array}$ \\
\hline Chap. VII : Facteurs qualitatifs (4) & Chap. VII : Facteurs qualitatifs (4) & Chap. VII : Facteurs qualitatifs (4) \\
\hline Deuxième partie & Deuxième partie & Deuxième \\
\hline Résultats généraux (1) & Résultats généraux (1) & Données de fait (2) \\
\hline Chap. I : État des Populations (6) & Chap. I : État des Populations (7) & Chap. I : État des populations (7) \\
\hline Chap. II : Mortalité (5) & Chap. II : Mortalité (5) & Chap. II : Mortalité, morbidité (5) \\
\hline Chap. III : Natalité (7) & Chap. III : Natalité, fécondité (7) & Chap. III : Natalité, fécondité (6) \\
\hline $\begin{array}{l}\text { Chap. IV : Mouvement général et } \\
\text { vitalité (5) }\end{array}$ & $\begin{array}{l}\text { Chap. IV : Mouvement général et } \\
\text { vitalité (5) }\end{array}$ & $\begin{array}{l}\text { Chap. IV : Mouvement général et } \\
\text { vitalité (4) }\end{array}$ \\
\hline $\begin{array}{l}\text { Chap. V : La population française } \\
\text { (14) }\end{array}$ & $\begin{array}{l}\text { Chap. V : La population française } \\
\text { (13) }\end{array}$ & $\begin{array}{l}\text { Chap. V : La population française } \\
\text { (12) }\end{array}$ \\
\hline Troisième partie & Troisième partie & Troisième partie \\
\hline Doctrines de population (2) & $\begin{array}{l}\text { Doctrines et politiques de popu- } \\
\quad \text { lation (2) }\end{array}$ & $\begin{array}{l}\text { Doctrines et politiques de popu- } \\
\text { lation (2) }\end{array}$ \\
\hline $\begin{array}{l}\text { Chap. I : Doctrines classiques et } \\
\text { doctrines virtuelles (7) }\end{array}$ & Chap. I : Bref historique (4) & Chap. I : Bref historique (4) \\
\hline $\begin{array}{l}\text { Chap. II : Bases d'une doctrine } \\
\text { positive (12) }\end{array}$ & $\begin{array}{l}\text { Chap. II : Doctrines actuelles et } \\
\text { attitudes (4) }\end{array}$ & $\begin{array}{l}\text { Chap. II : Doctrines actuelles et } \\
\text { attitudes (4) }\end{array}$ \\
\hline \multirow[t]{3}{*}{$\begin{array}{l}\text { Chap. III : Politique et population } \\
\text { (7) }\end{array}$} & $\begin{array}{l}\text { Chap. III : Bases d'une doctrine } \\
\text { rationnelle (10) }\end{array}$ & Chap. III : Notion d'optimum (8) \\
\hline & Chap. IV : Le Tiers Monde (6) & $\begin{array}{l}\text { Chap. IV : Les problèmes Actuels } \\
\text { (10) }\end{array}$ \\
\hline & $\begin{array}{l}\text { Chap. V : Politique de Population } \\
\text { (5) }\end{array}$ & $\begin{array}{l}\text { Chap. V : Politique de Population } \\
\text { (5) }\end{array}$ \\
\hline Bibliographie (1) & Bibliographie sommaire (1) & Bibliographie (1) \\
\hline Table des matières ( 1 ) & Table des matières (2) & Table des matières (3) \\
\hline
\end{tabular}

Les remerciements placés en tête de certains ouvrages permettent d'identifier des collaborateurs de Sauvy tels que l'économiste Anita 
Hirsch, Frédéric Tabah ${ }^{12}$ qui ira ensuite au BIT, Georges Malignac venu de l'Insee et la sociologue Cécile Gotchac. Le démographe Jean-Claude Chesnais et le géographe Alain Lefebvre furent plus tard des collaborateurs directs de Sauvy. Mais rien dans les différentes éditions de ce «Que-sais-je ?» ne permet de déceler l'apport de tel ou tel collaborateur. La réédition posthume de 1992 pourrait avoir été gérée par Anne Sauvy, sa fille, avec l'aide de Jean-Claude Chesnais. L'édition de 1992 est en fait l'exacte reproduction de celle de 1984 (avant dernière édition), le seul ajout étant en bibliographie la référence au «Que-sais-je ?» La démographie publiée entre temps par Chesnais (1990).

Dans la première partie de la première édition de La population, Sauvy insiste sur la collecte des données de base et sur les mesures statiques, permettant de caractériser l'état d'une population (répartition par âges, par état matrimonial, par lieu de naissance, etc.) et celles dynamiques ou plus exactement «cinématiques» - qui permettent d'en décrire le mouvement, c'est-à-dire «tout changement apporté à l'état de la population». Les phénomènes en jeu (mortalité et natalité) sont examinés. Sauvy consacre un chapitre aux prévisions démographiques et un autre aux «facteurs qualitatifs» parmi lesquels figurent aussi bien les données concernant l'état de santé d'une population (statistiques de morbidité) que le poids et la taille des individus.

La deuxième partie de l'ouvrage fournit des données factuelles aux échelles mondiale et française. Dans la troisième partie sont discutées les notions de population maximale et d'optimum de population, de surpopulation et de sous-population, de même que les théories populationniste et malthusienne.

$\mathrm{Au}$ fil des éditions, les différents chapitres évoluent mais sans que la structure générale soit affectée. La table des matières de l'édition de 1992 ressemble singulièrement à celle de 1944, mais l'accent est davantage mis sur les «problèmes actuels» comme le vieillissement de la population.

\section{La démographie, trop méconnue}

Dans La population, comme dans de nombreux autres ouvrages, Sauvy qualifie la démographie de science ou discipline qui reste bien mal con-

12. Frédéric Tabah était le frère de Léon Tabah, directeur de la Division de la population des Nations Unies de 1972 à 1984. 
nue, alors que les phénomènes de population jouent un rôle majeur dans l'histoire des sociétés. Évoquant une faible assistance à son cours du Collège de France, il expliquait qu'il n'y avait «aucune chance [d'un large auditoire] pour la démographie, tant la population ignore la population»: bien que les individus soient très concernés dans leur vie personnelle par les changements démographiques, ils n'en sont pas conscients (Sauvy, 1981).

Toutes les éditions successives de La population, de 1944 à 1992, font état du nombre insuffisant de démographes en France ; Sauvy se réjouit cependant, dans la deuxième édition (1948), de la création de l'Institut national d'études démographiques (Ined). Dans la quatrième édition (1957), il regrette toujours qu'il y ait trop peu de démographes «dans la plupart des pays» ; dans l'édition de 1984, dernière à être publiée de son vivant, son constat est le même.

Pour Sauvy, non seulement les pays manquaient de démographes mais la discipline elle-même ne s'institutionnalisait pas véritablement. La préface de l'ouvrage De Malthus à Mao Tsé-Toung se termine par une réflexion d'ordre épistémologique sur le champ de la démographie, ce qui n'est pas habituel chez Sauvy :

«Le domaine de la population comprend des terrains étrangement sûrs et de redoutables mystères. Nous nous sommes efforcé d'éclairer ce qui peut l'être, y compris les redoutables points d'interrogation sans lesquels il n'y aurait plus de véritable science, faute de frontières» (Sauvy, 1958).

L'idée principale et récurrente était celle d'une «science sauvage». Ainsi, dans l'avant-propos du volume I de la Théorie générale de la population, il affirmait que «[c]ette science sauvage qu'est la démographie, sans maîtres ni élèves, [devait] être cultivée à sa place» (Sauvy, 1963). Par "cultivée à sa place», il signifiait que cette science méritait d'être pleinement reconnue, étant donné que «par de nombreux aspects [...] la recherche économique ou [...] la politique générale [venait] buter sur le problème de population». On ne pouvait par conséquent pas continuer «de négliger plus longtemps cette question, de la maintenir hors des programmes universitaires».

Le succès de La population, et le fait que cet ouvrage ait été réédité à de nombreuses reprises, a pu faire dire qu'il s'agissait d'un «classique de la démographie» (Armengaud, 1971). On y trouverait une sorte de codification de ce que le terme "population» peut recouvrir, un peu à la manière de Roland Pressat, désireux il y a plusieurs décennies de fixer le champ, le vocabulaire et les méthodes d'une analyse démographique alors en pleine évolution ou de Louis Henry préparant la deuxième édition française du Dictionnaire démographique multilingue (UIESP, 1981). 
Dans un texte paru en 1971 dans la rubrique «bibliographie critique» de la revue Population, l'historien André Armengaud voit dans la succession des rééditions de ce petit ouvrage les signes d'une science qui se consolide :

«La première édition de ce petit livre, devenu un classique de la démographie, remonte à 1944. Voici que vient de paraître la 10ème édition, soigneusement mise à jour, à l'aide des chiffres les plus récents. Elle prouve que la démographie a cessé d'être 'la science sauvage, sans maître, ni élève' dont S. dénonçait naguère l'injuste destinée» (Armengaud, 1971).

Cependant, dix ans plus tard, à l'heure des bilans, Sauvy estime que «la démographie reste bien alors la science sauvage qui ne s'enseigne pas» (Sauvy, 1981). Une des raisons pour expliquer le peu de formations en démographie tiendrait selon lui au fait que, contrairement à ce qu'il en est pour d'autres disciplines, les objets de la démographie sont familiers (naissances, décès, migrations, etc.) : il est dès lors plus difficile de convaincre l'opinion publique (et des décideurs politiques ou administratifs) qu'il s'agit véritablement d'une science ${ }^{13}$. Pour ce qui est de l'enseignement proprement dit, c'est sans doute par habitude que Sauvy continuait à la fin de sa vie de déplorer que la démographie ne soit guère enseignée, car il existait à ce moment-là bien des formations spécialisées en France (en particulier à Paris, Bordeaux et Strasbourg) comme à l'étranger (à Louvain-la-Neuve et à Montréal, pour se limiter à des enseignements francophones), tandis que la démographie faisait souvent partie du cursus des études universitaires de sciences économiques ou de sociologie par exemple.

\section{Des phénomènes majeurs mais peu visibles}

Inventeur du terme «démographie», Achille Guillard $(1855,2013)$ considérait cette discipline comme un «piédestal solide» sur lequel s'appuient «les sciences économiques et sociales». Cette idée que tout relève à l'origine de la population se retrouve chez Sauvy. La dimension démographique du changement des sociétés est non seulement jugée majeure mais aussi, en quelque sorte, «première». La démographie s'intéresse à des phénomènes de population lents, peu visibles mais qui seraient dé-

13. Dans l'hommage qu'il rend au Collège de France à Alfred Sauvy après sa disparition, l'économiste Edmond Malinvaud, qui y est alors professeur, note à son propos que «[c]ette conception d'une science aux frontières mal définies lui plaisait» (www. college-de-france.fr/site/professeurs-disparus/alfred_sauvy.htm). 
terminants. Dans sa Leçon inaugurale au Collège de France en 1959, il déclarait ne pas souhaiter s'arrêter aux «phénomènes de surface», citant à cette occasion Paul Valéry: «Les événements m'ennuient. Les événements ne sont que l'écume des choses, ce qui m'intéresse, c'est la mer». Dans la première édition de La population, en tout début d'introduction, il insiste sur cette lenteur et par voie de conséquence faible visibilité des phénomènes démographiques :

«La lenteur des phénomènes démographiques les charge de conséquences, tout en les dérobant à l'attention des contemporains qui les subissent. La plupart des événements historiques profonds trouvent leur explication dans des phénomènes peu connus de population» (LP 1944, p. 5).

Dans la quatrième édition de l'ouvrage, parue en 1957, il introduit la fameuse comparaison entre vitesse des phénomènes démographiques et mesure du temps :

«La petite aiguille de la montre est la plus importante, mais elle paraît immobile» (LP 1957, p. 5).

L'importance de la démographie comme discipline ne se perçoit véritablement que dans le temps long. Pour appuyer cette affirmation, dans cette même édition de 1957, Sauvy lie l'expansion de la langue anglaise en Amérique du Nord aux migrations différentielles : pendant un siècle et demi les «bateaux anglais ont amené chaque année des milliers d'immigrants [...] tandis que les bateaux français n'en apportaient que quelques centaines» (LP 1957, p. 5).

Sauvy tire les conséquences de l'inertie démographique : «[...] les mouvements démographiques étant lents, les modifications qui leur sont données doivent être plus lentes encore» (LP 1944, p. 113). Dans la gestion des évolutions démographiques (baisse de la natalité par exemple), «[u]n coup de frein trop brusque entraîne diverses secousses» ${ }^{14}$ écrit-il, ce qui prouve certes qu'il se préoccupait d'éclairer l'action mais qu'il était ipso facto partisan d'une action pour corriger des mouvements de population qui ne seraient pas jugés satisfaisants, telle qu'une baisse marquée de la natalité à l'origine d'un intense vieillissement démographique.

14. On se souvient de l'article de Jean Bourgeois-Pichat et Si Ahmed Taleb (1970) montrant que le passage d'une situation de croissance démographique rapide à une croissance zéro immédiate générait des fluctuations de la structure par âge et de la fécondité «insoutenables». 


\section{L'«observation rationnelle» d'une société : ambition ou illusion ?}

Les objets de la démographie étant familiers et susceptibles a priori d'être considérés sans la moindre technicité, les erreurs d'analyse ou d'interprétation sont fréquentes. Sauvy pointe ces erreurs, de même que les illusions et les idées reçues. Il s'insurge aussi contre un «refus de voir», qu'il estime commun au monde politique et au grand public. On retrouve dans La population, clairement exposé, un thème très cher à son auteur : la large ignorance «des faits reconnus et des lois établies» qui tient en partie à des "préjugés tenaces».

L'insuffisance - «l'indigence»- des données est en partie responsable «des contradictions et des erreurs» (LP 1944, p. 124). S'il existe un besoin d'analyses plus approfondies, la nécessité de données complémentaires se fait cruellement ressentir. Sauvy note le faible intérêt pour les questions démographiques, tant de la part des pouvoirs publics que de la population en général :

\footnotetext{
«C'est à cette carence [méconnaissance des 'données essentielles'], particulièrement remarquable en France, qu'est due la stérilité de discussions qui devraient faire éclater la lumière» (LP 1944, p. 9).

«La connaissance de ces quelques données strictement arithmétiques [sur la fécondité] épargneraient bien des controverses stériles» (LP 1944, p. 95).

«Comme en matière économique, les progrès importants [des sciences de l'homme] ne peuvent plus découler que d'une stricte observation des faits. Or, il en est peu qui soient moins connus, moins diffusés, que ceux qui se rapportent aux questions de population» (LP 1944, p. 99).
}

L'édition de 1984 (par conséquent aussi celle de 1992) dénonce le simplisme et la charge d'affectivité dans les débats entre malthusiens et populationnistes. Elle déplore «l'erreur générale des modèles et des théories : ignorer le facteur humain et la réaction de l'homme devant la difficulté». Sauvy oppose «l'observation rationnelle» de la société à «une observation superficielle et souvent affective». Croire que les emplois existent en quantité limitée, et même que leur nombre diminue, croire que les actifs doivent se partager ces emplois, en réduisant la durée du travail et en abaissant l'âge de la retraite, ne saurait ainsi relever de l'observation rationnelle des dynamiques de l'emploi.

Si l'idée qu'elle illustre - celle d'une inadéquation entre structure de l'offre et de la demande de travail - est bien là, la métaphore du gant n'est pas présente, bien qu'elle ait été introduite dès 1946 et que Sauvy y ait 
encore fait référence plus de trente ans plus tard, dans La Tragédie du pouvoir (1978) :

«Quand une main entre dans un gant, il arrive que l'étroitesse du gant empêche les doigts d'entrer dans leur logement, laissant des poches inoccupées. Le remède consiste, dans ce cas, à élargir le gant et non à couper les doigts... comme on le fit en 193715» (Debré, Sauvy, 1946).

«Pour entrer une main dans un gant, il faut un ajustement parfait de la forme ('il vous va comme un gant'). Supposons un marchand disant à une cliente : 'Madame ce gant doit vous aller parfaitement puisque son volume en centimètres cubes (je l'ai mesuré) est exactement égal à celui de votre main'. Cet homme serait jugé sévèrement. C'est cependant ce qui est fait constamment pour l'emploi ${ }^{16 » ~(S a u v y, ~ 1978) . ~}$

Toutes ces erreurs communes, comme il l'écrira ailleurs, sont largement véhiculées par les médias : «les illusions d'optique sociale les plus nocives, les ignorances les plus délétères, sont reprises [par les médias] avec la conscience d'un copiste médiéval» (Sauvy, 1981).

Mais l'observation que conduit Sauvy est-elle vraiment si «rationnelle»? Les jugements de valeur sur ce que doit être le niveau de la natalité ou l'effectif de la population par exemple ne sont jamais vraiment très loin. Pour tendre vers une observation rationnelle de la société, il lui aurait fallu jeter les bases d'une épistémologie de la démographie, ce qu'il ne fait en aucun cas.

\section{Les structures démographiques et leur dynamique}

Une analyse des structures de population à un instant donné permet de considérer les poids relatifs de différents sous-groupes ou de sous-populations. Les phénomènes étant dynamiques, les structures peuvent porter en elles un véritable potentiel, comme c'est le cas lorsqu'une population a enclenché un processus de vieillissement.

Sauvy s'intéresse aux structures selon l'âge ou l'état matrimonial mais aussi à celle que constituent les proportions de personnes vivant seules aux différents âges (Figure 1). L'instruction ou la profession, le caractère urbain ou rural de l'habitat, la nationalité française ou étrangère, etc. définissent également des structures.

15. Allusion à la réduction par le gouvernement du Front populaire de la semaine de travail de 45 à 40 heures.

16. En italique dans le texte. 
Figure 1 Exemples de structures démographiques présentées dans diverses éditions de La population

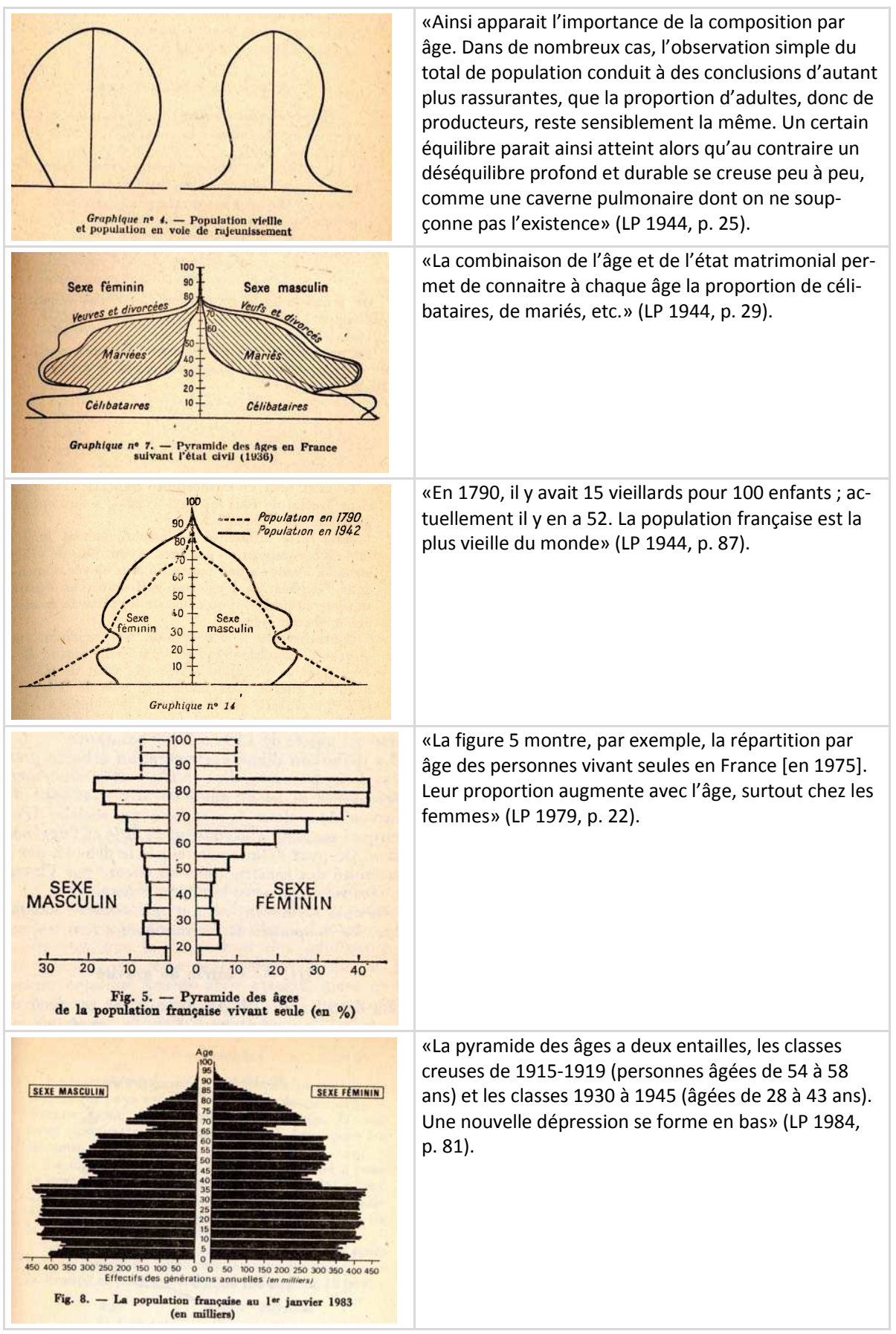


Naturellement, de nombreux développements portent sur la structure par âge, susceptible d'être révélatrice d'un problème démographique. Ainsi en est-il du vieillissement de la population marqué en France dès 1944 et qui inquiète déjà beaucoup Sauvy. La pyramide des âges de la population française vivant seule établie à partir des données du recensement de 1975 illustre de fortes différences entre hommes et femmes face à la solitude. La structure par âge de la population française en 1983, présentée dans la quatorzième édition de La population (celle de 1984), est encore profondément marquée par deux «entailles» dont celle des classes creuses des années 1915-1919. Le rétrécissement de la base de la pyramide est interprété comme «une nouvelle dépression».

Une autre approche de la structure d'une population privilégie la qualité. Dans un chapitre de la première édition consacré aux «facteurs qualitatifs», Sauvy mentionne l'état de santé, la situation d'infirme, le poids, la taille et diverses mensurations. Il fait état des tests d'intelligence permettant une mesure du quotient intellectuel. Dans l'édition de 1953, il est fait état de «tests caractériels» et, dans celle de 1957, de l'«assimilation culturelle des immigrants», qu'il est possible de «ranger aussi dans les problèmes qualitatifs».

S'ils se prêtent moins à la mesure, les facteurs qualitatifs ne sauraient pour autant être négligés, comme Sauvy entend le montrer à propos du cas français. Quelques questions soulevées dès l'édition de 1944 sont révélatrices de son état d'esprit :

\footnotetext{
«La réduction des naissances a-t-elle eu pour contre-partie une amélioration raciale, physique, intellectuelle?

L'infériorité quantitative de la France vis-à-vis des autres pays, a-t-elle été compensée par une supériorité qualitative?

L'amélioration raciale physique pourrait se constater à des caractéristiques telles que poids, taille, etc.» (LP 1944, p. 97).
}

Cette idée de compensation est reprise deux ans plus tard dans le texte signé conjointement avec Robert Debré : «constate-t-on que notre peuple a compensé son infériorité quantitative par un gain de qualité ?» (Debré, Sauvy, 1946). Dans les trois premières éditions (1944, 1948 et 1953) il est bien question d'«amélioration raciale, physique, intellectuelle» mais, à partir de la suivante (1957), on ne parle plus que d'«amélioration physique, intellectuelle».

Dans le chapitre consacré aux facteurs qualitatifs, il est aussi question de l'alcoolisme, auquel un chercheur de l'Ined a consacré deux volumes de la collection «Travaux et Documents» à la fin des années 1950 et au début des années 1960 (Ledermann, 1956, 1964), ainsi que d'une amélio- 
ration du niveau intellectuel de la population, mise en évidence par la baisse séculaire du nombre de conscrits illettrés. Dans les deux dernières éditions de La population, les considérations sur les facteurs qualitatifs sont plus brèves mais Sauvy note cependant que "[a]ucune amélioration relative de la population n'avait été observée, ni physique, ni morale, ni culturelle» ${ }^{17}$ (LP 1992, p. 89).

\section{Les outils du démographe : une présentation détaillée}

En dépit d'un volume imposé limité, l'ouvrage introduit les principaux outils du démographe. Ainsi, dès 1944 il est question de table de mortalité, de table de survie, de taux de mortalité par âge, de la méthode de la population type pour comparer des niveaux de mortalité ainsi que de celle de la mortalité-type. Il est question aussi de vie moyenne ou espérance de vie et de vie probable ou vie médiane. Le mode de calcul de l'espérance de vie est présenté et la possibilité de calculer des espérances de vie aux différents âges est mentionnée. Il est fait état de l'égalité entre le taux brut de mortalité et l'inverse de l'espérance de vie à la naissance dans «une population qui aurait pour composition par âges la table de survie et pour taux de mortalité à chaque âge ceux de la population étudiée», (LP 1944, p. 35). Il est également fait état des différences de mortalité selon la profession (avec intervention possible d'un effet de sélection, les personnes les plus fragiles choisissant des métiers plus sains, ce qui peut contribuer à une mortalité supérieure à ce qu'elle aurait pu être dans ces professions), de causes de décès (la question de la nomenclature est abordée) et de variation saisonnière de la mortalité. Dans l'édition de 1957 (4ème), Sauvy ajoute deux nouveaux paragraphes, l'un portant sur la mortalité infantile et l'autre sur la mortinatalité ; il introduit par ailleurs la notion de «mortalité du moment». L'économie générale du chapitre consacré à la mortalité ne sera plus modifiée.

Le chapitre sur la natalité introduit le taux de natalité générale, le taux de fécondité générale, la table de fécondité, le taux brut de reproduction ou taux brut de Kuczynski, les taux de fécondité légitime, etc. Des paragraphes sont consacrés à la légitimité des naissances et au nombre d'enfants par mariage (statistiques des familles), à la nuptialité et à la divortialité (tables de nuptialité, nombre de divorces), aux naissances suivant le sexe (masculinité), au mouvement saisonnier et à l'observa-

17. Cette phrase figure dans l'ouvrage en italique. 
tion continue de la natalité. Dans l'édition de 1948, le taux brut de reproduction devient aussi «taux de remplacement». En 1957, ce chapitre «Natalité» devient «Natalité, fécondité». Les probabilités d'agrandissement des familles, notion développée par Louis Henry, sont exposées. En 1961 (6ème édition), il est question de la fécondité d'une génération ou cohorte, de la fécondité annuelle, de la fécondabilité et de l'espacement des naissances. En 1975 (12ème édition), le paragraphe initialement intitulé «influence de l'âge» devient «influence de l'âge des parents et de l'âge au mariage». La distinction entre méthodes longitudinale et transversale est précisée :

«Comme pour la mortalité, on peut utiliser une méthode longitudinale ou une méthode transversale. La première consiste à suivre une cohorte composée, soit de femmes nées la même année, soit de femmes mariées pour la première fois, la même année. On obtient aussi, à la fin de la période de procréation, la descendance finale».

La descendance finale est différenciée du «nombre des naissances réduites», obtenu par la méthode transversale.

«Le mouvement général d'une population» fait l'objet d'un chapitre présentant le taux net de reproduction ou taux net de Kuczynski ainsi que les taux de stabilisation par la fécondité (inverse du taux net de reproduction) et taux de stabilisation par la mortalité $((\mathrm{T}-1) /(\mathrm{T}-\mathrm{t})$ où $\mathrm{T}$ est le taux brut et $t$ le taux net de reproduction). Il est aussi question de population stable, de population stationnaire et du taux de Lotka. La relation entre taux de Kuczynski et taux de Lotka est précisée :

Taux net de Kuczynski $=(1+\text { taux de Lotka })^{\text {Intervalle entre deux générations }}$

Dans l'édition de 1944, un paragraphe présente la population logistique. La sixième édition, celle de 1961, cite à son propos les noms de Verhulst et de Pearl. Le paragraphe consacré à la population logistique disparaît dans la 10ème édition parue en 1970, ainsi que dans les éditions ultérieures.

Dans l'édition de 1966, le paragraphe «Taux de Lotka» devient «Population stable et stationnaire». Sauvy introduit dans l'édition de 1968 un nouveau paragraphe consacré aux «Populations partielles», présentées comme fractions de la population vivant sur un territoire donné (population scolaire, population active, population retraitée, population médicale, etc.). Ce paragraphe est rebaptisé en 1970 : «Populations partielles ou sous-populations». Il y ajoute un alinéa :

\footnotetext{
«Deux populations partielles ou sous-populations peuvent vivre indépendantes l'une de l'autre (cas de deux religions, par exemple, en cas d'absence de mariage mixte) ou en dépendance étroite (population active et inactive par exemple)» (LP 1970, p. 46).
} 
Les fondements de l'analyse démographique sont donc présentés de manière assez complète dans La population, eu égard au format de l'ouvrage et au public visé. Mais il n'est fait pratiquement aucun cas de la recherche internationale, en particulier anglo-saxone.

\section{Un faible écho de la recherche internationale}

De manière pour le moins paradoxale, Sauvy a largement ignoré la recherche internationale, tant dans cet ouvrage que dans son œuvre en général. Il a pourtant participé aux réunions de la Commission de la population des Nations unies dès sa création ${ }^{18}$, ce qui lui donnait l'occasion de connaître les situations démographiques d'autres pays et les travaux des chercheurs étrangers. Il a parcouru le monde, de la Russie à Cuba, du Maroc où il rencontra Hassan II à l'Inde où il eut une entrevue avec Nehru, de la Tunisie où il fut invité par Bourguiba au Venezuela, etc., ce qui aurait dû l'inciter à privilégier une vision mondiale des questions de population. Bon nombre de ses ouvrages furent traduits dans différentes langues étrangères : la Théorie générale de la population fut ainsi traduite en anglais, en espagnol, en japonais et en chinois. Dans l'In memoriam paru dans Population and Development Review après la mort de Sauvy, Keyfitz le présentait comme «une figure mondiale dans les études de population durant les 50 dernières années» (Keyfitz, 1990). L'introduction du concept de «Tiers Monde» prouvait un intérêt de Sauvy pour les questions de population à l'échelle internationale. En dépit de tout cela, Sauvy a centré ses réflexions sur le seul cas français et n'a pas facilité l'accès de ses lecteurs à la recherche démographique mondiale.

On peut s'étonner, alors que Sauvy présente le taux de Lotka, qu'il ne fasse pas figurer en référence bibliographique la deuxième partie de la Théorie analytique des associations biologiques de Lotka (1939), parue en français, texte pourtant fondamental qui jette les bases de la dyna-

18. La Commission de la Population fut instituée par la résolution 3 du Conseil économique et social de l'Organisation des Nations Unies, en date du 3 octobre 1946. La première séance de la Commission se déroula du 6 au 19 février 1947 à Lake Success, sur l'île de Long Island (État de New York), où s'était établie l'ONU avant son installation définitive à Manhattan. «Douze hommes, de doctrines, de cultures et de pays différents, sont réunis autour d'une table, avec mission de définir... ce qu'ils auront à faire», raconte Sauvy (1981) à propos de cette première séance de la Commission de la population. Il y siègera jusqu'en 1974. Notons que, par une résolution du 19 décembre 1994, l'Assemblée générale décide de renommer la Commission de la population en «Commission de la population et du développement». 
mique des populations. Il ignore a fortiori des ouvrages de référence en langue anglaise comme The Growth and Structure of Human Populations. A Mathematical Investigation, publié par Ansley J. Coale au début des années 1970 (Coale, 1972).

Pour mettre en évidence cette faible attirance de Sauvy pour les recherches démographiques menées ailleurs qu'en France, comparons l'édition de 1970 de La population et La démographie que Jean BourgeoisPichat fait paraître un an plus tard (Bourgeois-Pichat, 1971). Dans une bibliographie certes qualifiée de «sommaire», Sauvy ne cite que 23 références : toutes concernent des publications en français. A contrario le livre de Bourgeois-Pichat ${ }^{19}$ cite quelque deux-cents ouvrages et articles, dont plus de la moitié en langue anglaise. Des publications en italien et en espagnol sont aussi mentionnées. Dans les Éléments de démographie, sorte d'approfondissement de La population en près de 400 pages (Sauvy, 1976), 128 ouvrages ou articles sont cités. Quelques auteurs anglophones traduits en français figurent dans la bibliographie (Berelson, Caldwell, Mc Namara, etc.) et deux références seulement sont en langue anglaise : Population Growth and Land Use de Colin Clark (1967) et un texte publié par les Nations unies.

Le même exercice de comparaison entre ouvrages d'initiation aux questions de population peut être réalisé en rapprochant l'édition de 1973 (11ème édition) de La population et le petit livre Comprendre la démographie que font paraître cette même année Hubert Gérard et Guillaume Wunsch (Gérard, Wunsch, 1973). Le contenu comme la bibliographie de ces deux ouvrages méritent d'être comparés. Ainsi le chapitre que Gérard et Wunsch consacrent à l'«évolution de la population mondiale» distingue clairement ce qui relève des faits (accélération de la croissance démographique ), des théories (modèle de la transition démographique) et des relations observables (corrélation entre espérance de vie à la naissance et revenu par tête) de ce qui constitue «le problème démographique contemporain», à savoir «une croissance trop rapide» de la population mondiale. La bibliographie de cet ouvrage est aussi plus conséquente et plus internationale. La comparaison entre deux «Que-saisje ?», la 15ème édition de La Population (1992) et la première édition de La démographie de Jean-Claude Chesnais (1990) montre plus d'ouverture internationale chez ce dernier : dans la liste des 14 ouvrages de la «bibliographie indicative», figure la deuxième partie de la Théorie analytique des associations biologiques (Lotka, 1939) ainsi que 3 ouvrages en langue anglaise, dont Applied Mathematical Demography (Keyfitz, 1985).

19. Jean Bourgeois-Pichat fut directeur de l'Ined de 1962 à 1968. 
Les 25 références de l'ouvrage de Sauvy ne renvoient qu'à des textes en français. Il est vrai que l'entrée par «la démographie» diffère en principe de celle par «la population» mais, dans les faits, les deux ouvrages présentent des similitudes.

Dans ses souvenirs, Sauvy explique ce repli sur la France en faisant état d'une «peur d'être déracinée» (Sauvy, 1981). La défense de la langue française passait pour lui par un rejet de l'anglais ; il voyait dans la domination internationale de cette langue et dans l'attrait qu'elle pouvait exercer auprès des Français une «double capitulation».

\section{La prévision, au cœur de la science de la population}

Pour Sauvy «la démographie est presque inséparable de la prévision», puisque «les perspectives donnent une base solide à la politique démographique». On retrouve indirectement cette idée que la science doit déboucher sur l'action. Ultérieurement, il considérera que les projections démographiques sont indispensables «à toute politique économique à long ou moyen terme».

Dans un chapitre de l'édition de 1944 consacré aux «prévisions démographiques», il détaille la méthode utilisée, s'appuyant sur l'observation des populations du passé, sur un choix d'hypothèses et sur la mise à jour de régularités, de constances, d'invariances (de nature simple ou en termes d'évolutions proportionnelles) ou sur la découverte d'une «loi déterminée» que suivrait une grandeur (le taux de mortalité par exemple).

Il est bien précisé qu'il s'agit de prévision conditionnelle et non de «prédiction». Il s'agit seulement de s'interroger sur ce qui se passerait si différentes hypothèses étaient vérifiées. Mais selon Sauvy, si le résultat obtenu n'apparaît pas satisfaisant (vieillissement projeté trop marqué par exemple) des politiques démographiques doivent être mises en place. La formule «prévoir pour ne pas voir» chère à Sauvy résume bien l'utilité des perspectives démographiques. Un parallèle imagé lui permet d'illustrer son propos :

«Lorsqu'un navire avance sur un écueil, la vigie signale cet écueil [,] ce qui revient à annoncer que si la direction n'est pas modifiée, le navire va sombrer. Mais loin de souhaiter ce naufrage, l'observateur travaille au contraire à l'éviter» (LP 1944, p. 56).

Pour éviter toute ambiguïté, dans l'édition de 1961 (6ème) l'intitulé du chapitre passe de «Prévisions démographiques» à «Projections, perspectives démographiques». Sauvy présente également des perspectives dé- 
rivées, comme celles relatives à l'évolution de la population active. La «population en âge ou en état d'être active» est ainsi obtenue en deux temps :

- calcul de la population totale par âge ou groupe d'âge,

- application à chaque nombre obtenu du taux d'activité par âge ou groupe d'âge correspondant.

Ces taux d'activité peuvent être supposés constants ou évoluer dans un sens prédéterminé. Il est aussi possible d'établir des perspectives pour la population agricole, de même que pour la population des élèves ou celles des maîtres.

$\mathrm{Si}$ «la démographie est presque inséparable de la prévision», en dépit de l'inertie des phénomènes, la prévision reste un exercice périlleux, particulièrement pour ce qui est de la fécondité. Ainsi Sauvy fut l'auteur, au début des années 1930, de projections démographiques, pour la France, à l'horizon 1980 (Sauvy, 1932). Deux hypothèses de fécondité étaient envisagées ${ }^{20}$ : une constance par rapport à la valeur de 1929-1930 (indice conjoncturel un peu supérieur à 2 enfants par femme) et une fécondité égale à celle du département de la Seine, nettement plus basse, ce qui conduisait à une population en 1980 de 38,9 millions dans un cas et de 29 millions dans l'autre, contre une population initiale de 40,7 millions en 1929 (l'effectif de la population française fut de 53,7 millions en 1980). Ces «calculs démographiques» furent démentis par une reprise durable de la natalité au lendemain de la Seconde Guerre mondiale. Sauvy éprouva d'ailleurs beaucoup de difficultés à admettre l'existence de cette reprise (Lévy, 1990). Il voyait dans la forte fécondité de la fin des années 1940 le rattrapage d'un «retard antérieur» tandis que Bourgeois-Pichat considérait «l'hypothèse d'un changement d'attitude des Français devant le fait 'naissance' [...] de plus en plus probable». Sans évoquer dans ses mémoires sa réticence à croire à une reprise quelque peu durable de la natalité, Sauvy déclare toutefois que «[c]'est sur les prévisions déjouées, que doivent porter les efforts... pour ébranler soimême la solidité de l'édifice laborieusement construit...» (Sauvy, 1981).

20. À propos de ces hypothèses, Sauvy écrivait : «[l]'Alliance Nationale [qui avait commandé ces perspectives] a choisi des hypothèses évidemment discutables, comme toutes les hypothèses concernant l'avenir, mais choisies de façon judicieuse. L'une est à dessein résolument optimiste et l'autre repose malheureusement sur des éventualités à redouter» (Sauvy, 1932). Il s'agissait, avec des hypothèses paraissant extrêmes, de construire un encadrement de l'avenir possible. Alors que la première hypothèse correspond à une constance des taux de fécondité par âge, dans la seconde, la fécondité diminue jusqu'à un palier correspondant au «niveau d'un département avancé comme la Seine», ce qui semble alors pouvoir préfigurer l'avenir du point de vue de la transition démographique. 
Le célèbre aphorisme «prévoir pour ne pas voir» souvent cité à propos de Sauvy et de la prévision, laisse bien entendre que dès lors qu'une évolution projetée n'est pas souhaitable, une politique doit être mise en œuvre pour la «corriger». L'élaboration de perspectives démographiques ne conduit donc pas seulement à éclairer l'action mais aussi à recommander une intervention.

\section{Un «équilibre» des populations peut-il exister?}

Les questions d'équilibres et de déséquilibres occupent une place importante dans La population, comme dans d'autres publications de Sau$v^{21}$. L'équilibre des populations primitives, rappelle-t-il, était la conséquence de famines, d'épidémies accompagnées de phénomènes de sousnutrition et également de guerres meurtrières. Il parle à ce propos d'équilibres naturels, la volonté humaine n'étant pas en jeu. La surpopulation était en réalité «combattue par les maux qu'elle engendrait». Elle pouvait aussi être combattue par la suppression des vieillards ou leur mise à l'épreuve physique (le haut de la pyramide des âges est alors seul affecté) mais également par l'infanticide, l'exposition des enfants, l'avortement ou les pratiques anticonceptionnelles (c'est dans ce cas le bas de la pyramide qui est affecté). Par ailleurs, dans une "population primitive», les plus faibles sont les plus touchés par la mortalité : une sélection s'opère.

Dans la première édition de La population, est introduit l'exemple - en réalité la «fable» ${ }^{22}$ - de l'Île de Juan Fernandez pour illustrer la régulation inter-espèces :

«Très instructif apparaît par exemple le peuplement de l'île de Juan Fernandez dans le Pacifique : dans cette île déserte, les Anglais introduisent un jour des chèvres pour s'assurer ultérieurement gibier et nourriture. Les chèvres se multiplient jusqu'à la limite permise par la superficie et la végétation de l'ile. Un peu plus tard, les Espagnols, par hostilité contre les Anglais, introduisent des dogues dans l'île. Ces dogues vivent aux dépens des chèvres et se multiplient à leur tour. Mais un équilibre s'établit bientôt entre les deux espèces, les chèvres

21. Dans Des Français pour la France, Debré et Sauvy (1946) parlent de «l'équilibre le plus important de tous, l'équilibre démographique» ajoutant que celui-ci ne saurait être confondu avec une quelconque «constance» ou «stagnation».

22. L'archipel Juan Fernandez est situé dans le Pacifique, au large du Chili. La «fable» des chèvres et des chiens est en réalité tirée de l'ouvrage $A$ Dissertation on the poor laws. By a well-wisher to Mankind que Joseph Townsend fit paraître à Londres en 1786. 
occupant les régions moins accessibles et ne faisant que de courtes incursions dans les parties basses» (LP 1944, p. 7).

Cette «fable» est supprimée dans l'édition de 1957, l'introduction étant considérablement raccourcie cette année-là. Toutefois, dans le cours Économie et population que Sauvy donnera à l'IDUP à un âge avancé (75 ans dépassés), il mentionnait encore cette fable qui lui tenait donc particulièrement à cœur.

Avec la «révolution démographique» que met en évidence Adolphe Landry en 1934, l'équilibre démographique n'est plus garanti, en raison de la restriction volontaire des mariages et des naissances. Une «politique consciente» est alors nécessaire pour rétablir l'équilibre souhaitable :

«L'équilibre, désormais artificiel, d'une population ne peut donc être assuré que par intervention de la puissance publique» (LP 1944, p. 119).

Trois temps sont en fait distingués :

- celui d'imprévoyance collective (jusqu'au 19ème siècle),

- celui de prévoyance individuelle et d'imprévoyance collective (conséquence du développement de la contraception),

- celui de prévoyance collective (mise en place d'une politique appropriée).

La notion d'équilibre est bien de nature dynamique. Certains pays peuvent être en déséquilibre structurel (en «état de dépopulation») en dépit d'un excédent des naissances sur les décès, celui-ci tenant à une structure par âge transitoirement favorable à la natalité. La question de la contribution de l'immigration à la restauration d'un équilibre est posée en termes très simples que l'on pourrait qualifier de pour le moins technocratiques, l'hypothèse de stationnarité simplifiant considérablement la dynamique de l'équilibre considérée ${ }^{23}$ :

«[...] si un écart est constaté entre l'équilibre démographique (correspondant par exemple à une population stationnaire) et la situation réelle, et que l'on cherche à rétablir l'équilibre par l'immigration, on pourrait déterminer ainsi les nombres d'immigrants de chaque âge qu'il conviendrait de laisser entrer sur le territoire» (LP 1944, p. 52).

Quarante ans plus tard, dans l'édition de 1984 (14ème), Sauvy ajoute, dans le chapitre portant sur la politique de population, un paragraphe intitulé «Déséquilibre et immigration». Il y écrit :

«Une migration intense des pays surpeuplés vers les pays occidentaux à population décroissante et vieillissante est 'écrite' à long terme. Seule reste inconnue

23. Pour une autre approche de l'équilibre démographique assuré par un courant migratoire, voir la question des migrations de remplacement (United Nations, 2000). 
la façon (désordre ou organisation) dont se feront ces mouvements vitaux» (LP 1984, p. 121).

Après s'être longtemps inquiété d'une sous-natalité, Sauvy évoque la «menace» d'une population jeune en croissance très rapide, consécutive au baby-boom (Sauvy, 1981). Dans La montée des jeunes, il ne parlait pas de menace mais d'«enjeu» :

«Exceptionnel est l'enjeu. Si la France réussit à accueillir ses jeunes, à les armer pour la vie, elle bénéficie des puissants effets du rajeunissement» (Sauvy, 1959).

Cette question des déséquilibres est aussi traitée dans La population sous l'angle des "problèmes démographiques», comme on le verra ultérieurement.

\section{Un attachement à la théorie dépassée de l’optimum}

Dans la partie traitant des doctrines classiques, Sauvy consacre un paragraphe qu'il nomme "population maximum et population optimum». La «notion d'optimum» fera même l'objet d'un chapitre dans l'édition de 1979 (13ème). L'optimum relève pour lui d'un «juste milieu» entre situations de sous-peuplement et de surpeuplement.

Maximum et optimum de population sont clairement distingués. Sauvy renvoie à Cantillon et à sa prise en compte des besoins des populations. Il rappelle les fondements de la théorie de l'optimum qui s'appuie sur la combinaison de deux effets jouant en sens inverse : économies externes liés à la division du travail, loi des rendements décroissants. Il rappelle aussi que, pour Gini (1931), il existait plusieurs valeurs optimales dépendant de l'état de la technique. L'optimum de la civilisation agricole n'est pas le même que celui de l'ère industrielle.

Sauvy affirme que, selon une croyance largement répandue, l'optimum de population serait dépassé : ainsi le chômage résulterait pour certains d'un excès des naissances. Deux écueils seraient par conséquent à éviter : la «surpopulation» et la «sous-population» (LP 1944, p. 108). La Chine et le Moyen Orient seraient, par exemple, en situation de surpopulation en raison d'une densité et d'une natalité excessives.

L'objectif à atteindre pour une société donnée n'est pas la maximisation du nombre d'habitants mais la maximisation du bien-être des populations. De son côté Landry a soutenu l'idée que l'on pouvait vouloir maximiser le bien être total (produit du bien être individuel par l'effectif de 
la population) et non seulement le bien-être par tête (Landry, 1929). La théorie de l'optimum soulève en réalité de nombreuses questions : il faut tenir compte des ressources naturelles disponibles et de la technique. Le «progrès» peut avoir un coût, par exemple s'il est associé à l'exode rural qui accroît la charge des logements à construire alors que d'autres logements tombent en ruine. Le progrès technique peut en réalité élever ou abaisser l'optimum. Le rythme du progrès peut aussi être en partie commandé par la population : l'augmentation trop rapide rend difficile I 'investissement ; l'augmentation trop lente n'incite pas à innover («le fils unique n'a qu'à suivre la filière paternelle»). Sauvy conclut que l'accroissement de la population, dans des «limites raisonnables», facilite l'équilibre dynamique au sein de la société. Il parle de «croissance raisonnable» ou même de «variation raisonnable» de la population, à la baisse ou à la hausse (LP 1959, p. 108).

Il est étonnant que les développements concernant la théorie de l'optimum de population, très en vogue dans l'entre-deux-guerres mais qui n'est plus débattue dans les années 1980 et a fortiori 1990, aient été conservés dans les dernières éditions de La population.

\section{Le prisme des «problèmes de population» : le retour du politique ?}

L'initiation à la démographie passe aussi pour Sauvy par une présentation des principaux «problèmes» de population, tels que le vieillissement de la population ou la croissance démographique rapide. Dès la première édition de La population, Sauvy s'inquiète du vieillissement, faisant remarquer qu'il y avait 15 «vieillards» pour 100 enfants en 1790 contre 52 en 1942. "La population française est la plus vieille du monde» note-t-il alors (LP 1944, p. 87). Puis, dans la 4ème édition, apparaît le fameux dilemme «croître ou vieillir» (LP 1957, p. 109). Le vieillissement du corps électoral est par ailleurs mis en avant, ainsi que l'augmentation des charges liées au paiement des retraites. Il fait observer que le capital et les revenus «se déplacent» vers les âges élevés.

L'autre grand problème est la croissance démographique qui s'est accélérée. L'édition de 1953 (3ème) inclut, par rapport aux éditions précédentes, un nouveau paragraphe, de taille conséquente, sur les pays «sous-développés». Le problème provient dans ces pays de ce que la mortalité a fortement baissé alors que la fécondité est restée inchangée :

«Cet équilibre millénaire (assuré du reste par famines, guerres et épidémies) est aujourd'hui rompu: du fait de l'introduction de techniques médicales effi- 
caces (antibiotiques, D.D.T.), la mortalité a notablement diminué. [...] Comme la fécondité est restée au même niveau, le cercle fermé de la vie et de la mort est ouvert et les populations s'accroissent» (LP 1953, p. 117).

Il est étonnant que ne soit faite aucune référence à la théorie de la transition démographique ; l'expression n'est pas utilisée pour rendre compte d'un processus pourtant bien décrit par les démographes de Princeton et bien d'autres dans les années 1950, 1960 et 1970. Sauvy considère que ce problème de croissance démographique ne peut être posé comme "problème de la population mondiale», compte tenu du «cloisonnement actuel des nations» et de «la fermeture des frontières aux immigrés» 24 .

Les pessimistes pour lesquels «la terre est déjà surpeuplée», ce qui nécessite d'introduire le «birth control», sont opposés dans ce paragraphe aux optimistes qui «font confiance aux progrès de la science» (la terre pourrait selon certains d'entre eux nourrir 12 milliards d'habitants). Sauvy propose de poser, pour chaque pays, tout un ensemble de questions parmi lesquelles trois concernent la natalité :
«[...] Peut-on prévoir une baisse de la natalité ?
[...] Est-il recommandable de la préconiser et de la favoriser?
[...] Est-il possible de la favoriser ? Par quels moyens ?» (LP 1957, p. 118).

L'hypothèse de «quelque produit comestible assurant une stérilité temporaire sous un très faible volume et revenant à très bas prix» est évoquée, car elle changerait profondément la donne, tant dans ces pays «sous-développés» que dans les «pays évolués». Or des laboratoires américains et indiens travaillent dans cette direction.

Dans l'édition suivante, de 1957, ce paragraphe sur les pays sous-développés devient un chapitre à part entière. Il est au demeurant curieux qu'il ne soit fait à aucun moment référence au «Tiers Monde», alors que Sauvy a introduit cette expression en $1952^{25}$. Dans l'édition de 1959, il est fait allusion à la «pilule stérilisante». En 1963, à propos de cette dernière, il envisage la dimension santé du nouveau moyen contraceptif en cours d'élaboration, évoquant un «stérilisant temporaire sans nocivité et revenant à très bas prix».

24. Cette question est développée longuement par Alfred Sauvy dans «Le 'faux problème' de la population mondiale», Population, 3, 1949, pp. 447-462.

25. Alfred Sauvy, «Trois mondes, une planète», L'observateur, 118, 14 août 1952. 
Figure 2 Comparaison des modalités de reproduction dans des populations traditionnelle, évoluée et du Tiers Monde ${ }^{26}$
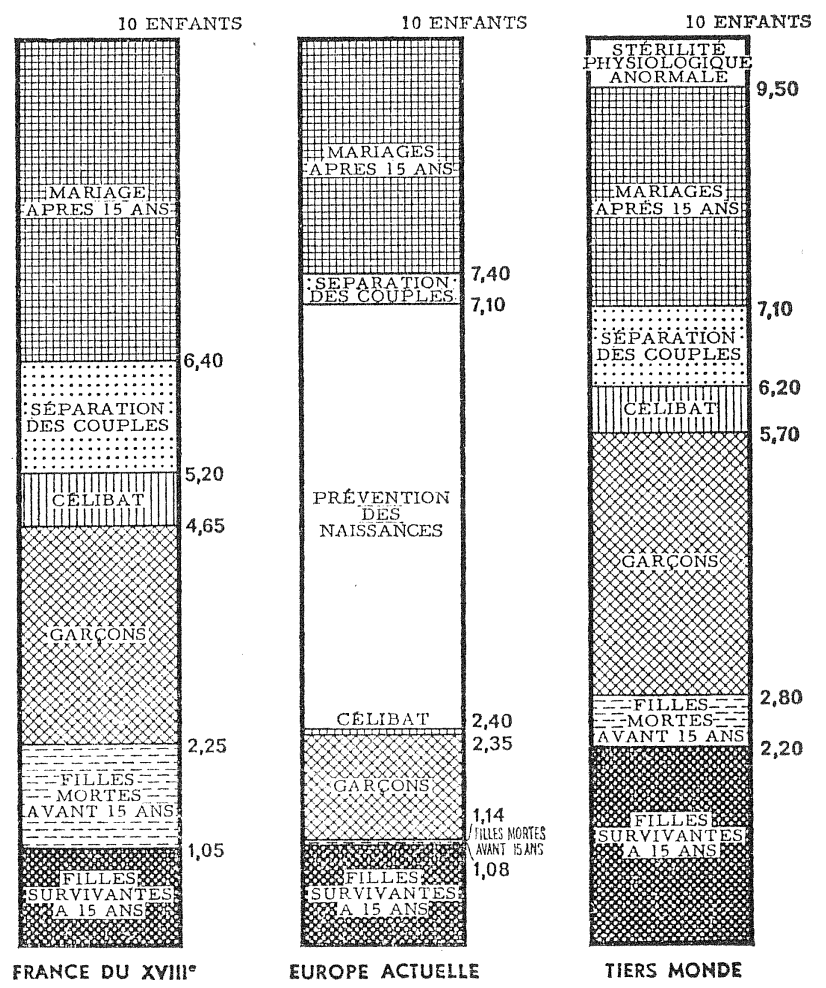

Fig. 10. - La reproduction des trois types de population : popu. lation traditionnelle (France du Xvirre siècle), population évoluée (Europe occidentale actuelle) et Tiers Monde.

Le chapitre «Les pays sous-développés» devient «Le Tiers Monde» dans la 8ème édition, datant de 1966. Une nouvelle figure est introduite, comparant les facteurs de la reproduction de «trois types de population», la France du 18ème siècle («population traditionnelle»), l'Europe actuelle («population évoluée») et le Tiers Monde (Figure 2) ${ }^{27}$. Les effets de la survie, de la nuptialité, de la contraception, etc. sur le nombre final d'enfants sont mis en évidence.

26. Schéma présenté dans les 9ème, 10ème et 11ème éditions de La population, parues respectivement en 1968, 1970 et 1973.

27. Cette figure disparaît de l'édition de 1975 (12ème). 
Il n'est plus seulement question de pilule stérilisante, mais également de «corps intra-utérins». Les différences entre répartitions de la population mondiale et du revenu mondial sont illustrées à l'aide d'une figure montrant qu'en 1965 le poids des pays peu développés est de $44,6 \%$ pour ce qui est de la population et de seulement $14,2 \%$ pour le revenu.

En 1968 (9ème édition de l'ouvrage), un premier bilan des nouveaux moyens contraceptifs est esquissé :

«Si la pilule stérilisante n'a guère modifié cette réponse [à la forte croissance démographique], par contre le stérilet (corps intra-utérin) change profondément la situation, par l'absence de contrainte continue qui caractérise ce procédé» (LP 1968, p. 119).

Le chapitre «Tiers Monde» devient «La population du monde» dans la 11ème édition, parue en 1973. Le texte est alors profondément remanié. Il est question d'un possible conflit entre riches et pauvres (menace que font peser les différences de répartition entre population mondiale et revenu mondial; consommation plus élevée des pays riches), de la croissance zéro, du rapport du MIT au Club de Rome ${ }^{28}$, des possibilités alimentaires, des ressources naturelles et de leur gaspillage. Dans l'édition de 1975 (p. 114), une distinction est faite entre deux formes d'inertie affectant les pays à croissance démographique rapide :

- une «inertie de caractère éthique et culturel»,

- une «inertie mécanique».

Sauvy fait état de ce que "[l]a Conférence mondiale de la Population à Bucarest a exalté avant tout la priorité du développement économique sur la prévention des naissances». Il reproche au Club de Rome d'avoir utilisé «des techniques en apparence rigoureuses» et ignoré l'importance du «facteur humain». Il cite Joseph Klatzmann $(1975,1983)$, pour qui la terre pouvait nourrir 10 milliards d'habitants.

En 1979 (13ème édition), le chapitre «La population du monde» disparaît au profit d'un nouvel intitulé : «Les problèmes actuels». Sauvy y aborde successivement le cas des pays peu développés, qui se caractérisent par une «croissance de population rapide» et une «économie attardée», celui des pays développés dont les sociétés manifestent un «refus

28. Meadows et al. (1972). Sauvy juge ce rapport avec sévérité : «[e]n dépit de ses légèretés, de ses inexactitudes, de ses complaisances, le rapport du Massachusetts I. T. au Club de Rome aura eu le mérite de poser la question en termes assez clairs» (LP 1973, p. 117). Sa critique dans l'édition suivante se veut radicale : «[l]oin d'éclairer la question, la méthode de la 'dynamique des systèmes' utilisée par le premier, puis le second rapport au Club de Rome [Pestel E., Mesarovic M. (1974), Stratégie pour demain, Paris, Seuil], l'obscurcit et la fausse, parce qu'elle laisse de côté, dans ses techniques en apparence rigoureuses, des facteurs importants, parmi lesquels le facteur humain». 
de la vie» (en ne se préoccupant pas des enfants) et qui doivent faire face au vieillissement démographique - un «conflit entre générations» paraît inéluctable aux yeux de Sauvy - et celui des pays socialistes où il peut y avoir, contrairement à la doctrine qui a eu longtemps cours, «surpopulation» (la Chine est citée). Le chapitre se termine par une «vue d'ensemble sur le monde».

Sauvy s'est obstiné à montrer l'absence de corrélation inverse entre croissance démographique et variation de la production agricole par habitant (ou PNB par habitant), afin de montrer que les discours alarmistes sur l'explosion démographique étaient sans fondement. «La réaction de l'homme devant la difficulté» (LP 1984, p. 111) - Sauvy ne cite pas Ester Boserup et la "pression créatrice» - peut en partie expliquer cette absence de corrélation négative. Ne cessant de s'opposer au courant néo-malthusien, Sauvy ne peut voir dans la croissance démographique mondiale la cause ou du moins un des facteurs de la crise mondiale de l'environnement. Il préfère accuser la production industrielle effrénée des pays riches, qui se traduit par des émissions de gaz carbonique en quantité toujours croissante.

\section{Matière à controverses}

Intitulé à partir de l'édition de 1953 «Politique de population», le chapitre terminal de La population est une occasion pour Sauvy d'aborder des questions de société controversées.

Un paragraphe traite de la famille qui a connu des vicissitudes depuis la fin du 18ème siècle : l'émancipation des femmes comme des enfants et le divorce auraient pu faire croire dans les années 1920 à «la fin du mariage [et à] l'élevage communautaire des enfants» (LP 1979, p. 120 et 121). La fin de la Seconde Guerre mondiale marque un renouveau de la famille, l'État accordant à celle-ci «des avantages ou des compensations». Mais la montée de l'illégitimité des naissances, du divorce, des unions hors mariages sont pour Sauvy une nouvelle menace pour l'institution familiale, d'autant plus que, depuis le début des années 1960, il constate un «encouragement aux pratiques anti-natales du reste devenues plus efficaces, [l']émancipation juridique et économique de la femme, [la] baisse en valeur réelle de l'aide à la famille» (LP 1992, p. 121).

D’autres questions sont évoquées dans l'édition de 1953 (Tableau 3) comme la sélection des meilleurs (la «stérilisation des tarés» est évoquée), l'euthanasie, le choix du conjoint («certaines incompatibilités en- 
tre conjoints [...] peuvent être nuisibles à la santé des enfants»), l'insémination artificielle et l'eugénique. En 1957 la «sélection des meilleurs» devient la «sélection biologique». Dans l'édition de 1973 (11ème), sont introduites trois nouvelles questions : la recherche de paternité qui se pose en raison de «récentes découvertes génétiques», la prolongation de la vie (et le coût qu'elle peut impliquer en termes de santé) et l'avortement. En 1979 (13ème édition), une distinction est introduite entre «euthanasie active (donner la mort)» et «euthanasie passive (ne pas donner tous les soins propres à maintenir la vie)». Sauvy précise que cette dernière est souvent opposée à «'l'acharnement thérapeutique' qui pose des problèmes humains et financiers». Dans la 14ème édition, publiée en 1984, le paragraphe «Famille» devient «Famille et natalité», sans changement notable de contenu. Un nouveau paragraphe «Déséquilibre et immigration» est introduit annonçant le caractère inéluctable des migrations des «pays surpeuplés» vers ceux «à population décroissante et vieillissante». Dans cette même édition, est évoquée l'économie de la santé qui prend de l'importance car si «la santé n'a pas de prix», elle a un coût.

TABLEAU 3 Nouveaux problèmes de société mentionnés par Sauvy dans le chapitre terminal de La population

\begin{tabular}{|c|c|c|c|c|c|}
\hline $\begin{array}{l}\text { Édition de } 1953 \\
\text { (3ème) }\end{array}$ & $\begin{array}{c}\text { Édition de } 1957 \\
\text { (4ème) }\end{array}$ & ... & $\begin{array}{c}\text { Édition de } 1973 \\
\text { (11ème) }\end{array}$ & $\ldots$ & $\begin{array}{l}\text { Édition de } 1984 \\
\text { (14ème) }\end{array}$ \\
\hline Famille & Famille & & Famille & & Famille \\
\hline $\begin{array}{c}\text { Sélection des } \\
\text { meilleurs }\end{array}$ & Sélection biologique & & Sélection biologique & & Sélection biologique \\
\hline Euthanasie & Euthanasie & & Euthanasie & & Euthanasie \\
\hline Choix du conjoint & Choix du conjoint & & Choix du conjoint & & Choix du conjoint \\
\hline $\begin{array}{l}\text { Insémination } \\
\text { artificielle }\end{array}$ & $\begin{array}{l}\text { Insémination } \\
\text { artificielle }\end{array}$ & & $\begin{array}{l}\text { Insémination } \\
\text { artificielle }\end{array}$ & & $\begin{array}{l}\text { Insémination } \\
\text { artificielle }\end{array}$ \\
\hline \multirow[t]{4}{*}{ Eugénique } & Eugénique & & Recherche de paternité & & Recherche de paternité \\
\hline & & & Prolongation de la vie & & Prolongation de la vie \\
\hline & & & Avortement & & Avortement \\
\hline & & & & & Économie de la santé \\
\hline
\end{tabular}

Cette introduction des problèmes démographiques dans un ouvrage de cette taille montre que Sauvy entend que les questions de société soient bien présentes dans le débat social. Par contre, il les aborde de manière plutôt allusive. Pour ce qui est de l'avortement provoqué, rappelons que Debré et lui considéraient en 1946 qu'il fallait le considérer comme «un mal social», à prévenir plutôt qu'à punir. La «lutte contre l'avortement» devait en réalité être une des composantes de la «lutte pour la natalité». 
Beaucoup plus tard, dans La Tragédie du pouvoir (1978), il se montre critique à l'égard de la loi sur l'Interruption volontaire de grossesse (IVG) de 1975, sans pour autant la nommer expressément :

«Faute de mesures favorables à la venue et à l'accueil de l'enfant, la loi sur l'avortement a eu le résultat logique : au lieu de devenir, au moins en partie, des grossesses désirées et des enfants accueillis, les grossesses non expressément voulues sont devenues des avortements, en attendant de devenir des regrets» (Sauvy, 1978).

Les dernières éditions de La population n'abordent cette question de l'avortement que d'une manière quasi factuelle :

«L'avortement a gagné du terrain dans les pays évolués, du fait du désir de libérer les femmes, des progrès des techniques abortives et des illusions répandues sur la parfaite efficacité des techniques contraceptives» (LP 1984, 1992, p. 124).

Quant à l'euthanasie légale, Sauvy s'y montre hostile même si «au vu des cas individuels» on pourrait être tenté d'y être favorable. La légalisation de l'euthanasie ne pouvait, selon lui, que conduire «aux abus les plus avilissants pour l'espèce humaine».

\section{Une sévère critique du mouvement écologiste}

Dans La population, comme dans d'autres publications et en particulier dans Croissance zéro ? (1973) où il se présente non seulement comme professeur honoraire au Collège de France mais aussi comme expert du Haut-Comité de l'Environnement, Sauvy se montre très critique à l'égard du discours écologiste. Il évoque dans Croissance zéro ?, une «crise de pessimisme morbide» en 1970, aux États-Unis, qui a conduit en particulier à la recommandation d'un arrêt de la croissance démographique (zero population growth), idée bien naïve à ses yeux. Il peut même montrer une franche hostilité à l'égard du mouvement et des thèses écologistes. Il affirme ainsi que «soucieux de préserver la nature, les écologistes redoutent les hommes» (Sauvy, 1978). Bien que lié personnellement à l'agronome René Dumont, connu pour son combat écologiste ${ }^{29}$, il écrit à son propos :

29. Dumont, favorable à une agriculture productiviste en début de carrière, participait au lendemain de la guerre avec Sauvy à des réunions préfigurant le Commissariat général au plan. 
«René Dumont est un homme de grand cœur, mais qui, comme la plupart des écologistes, ignore à un degré rare les rudiments de la science de la population» (Sauvy, 1978).

Pour montrer que les écologistes sont loin des réalités, il minimise par exemple le risque nucléaire affirmant que «la probabilité d'un accident tragique faisant 1000 morts est de l'ordre du milliardième» (Sauvy, 1978). Qu'il s'agisse des écologistes en général ou du Club de Rome en particulier, ses propos sont d'une rare sévérité :

«Et par ce même don de diplopie [vision dédoublée en présence d'un seul objet], les écologistes sont les plus ingénus de tous : ils condamnent le maudit nucléaire, en comptant sur le soleil et le vent, en pleine béatitude» (Sauvy,1981).

Quant au 1er rapport du MIT (Meadows), il est qualifié de «pseudo scientifique». Nous avions déjà noté par ailleurs que les préoccupations d'ordre écologique étaient absentes de la Théorie générale de la population, œuvre maîtresse de Sauvy. Les seules dimensions de la terre prises en compte étaient l'espace disponible et les ressources naturelles, en quantité limitée (Véron, 1992). En dépit de son titre, La terre et les hommes (1990), Sauvy fait dans ce livre état des prises de position inquiètes des dernières décennies sur les questions écologiques, non sans détachement.

\section{Une «dérive» idéologique en fin de vie ?}

À la fin de sa vie, Sauvy s'est montré proche des milieux conservateurs. La publication de L'Europe submergée. Sud-Nord dans 30 ans (Sauvy 1987) a pu accréditer la thèse d'une proximité avec l'extrême droite politique, même s'il ne semble pas qu'il ait eu de liens directs avec celle-ci. Toujours est-il que l'on ne peut que s'étonner, en examinant les bibliographies des dernières éditions de La population, de constater que des ouvrages classiques comme La révolution démographique de Landry (1934) ou Les avantages et les inconvénients économiques d'une population stationnaire de Stassart (1965) disparaissent au profit d'ouvrages essentiellement polémiques, dénonçant en particulier la dénatalité ou le vieillissement de la population. Dans la bibliographie des dernières éditions, figure ainsi La peste blanche (Chaunu et Suffert, 1976), La France ridée (Dumont et al., 1981), livre auquel Sauvy a lui-même collaboré et Les berceaux vides de Marianne (Biraben, Dupâquier, 1981). Seuls trois ouvrages classiques sont mentionnés jusque dans les dernières éditions: l'Histoire des populations françaises de Philippe Ariès (1948), l'Histoire de la population mondiale de 1700 à 1948 de Marcel Reinhard 
(1949), devenu Histoire générale de la population mondiale quand Reinhard collabore avec Armengaud (1961), puis avec Armengaud et Dupâquier (1968) et Le traité de démographie de Landry $(1945,1949)$. Placer en référence La peste blanche ne se justifie nullement, ce livre étant une dénonciation des évolutions démographiques alors en cours et principalement de la basse fécondité, dénonciation accompagnée de propositions pour éviter «le suicide de l'Occident». Mais, dans la première édition de La population, Sauvy citait bien La Résurrection par la natalité de Boverat (1942) ! Par contre, si les références changent à diverses reprises au fil des éditions pour laisser la place à des ouvrages plus politiques que scientifiques, l'économie générale du texte n'est aucunement modifiée à ces diverses occasions.

Certes Sauvy s'est montré de plus en plus proche des milieux conservateurs en avançant en âge, mais il l'avait déjà été à d'autres périodes de sa vie (Lévy, 1990 ; Rosental, 2003). Les prévisions qu'il avait réalisées au début des années 1930 lui avaient été «commandées» par l'Alliance nationale pour l'accroissement de la population française, milieu nataliste par excellence ${ }^{30}$. Proche de personnalités politiques de droite mais aussi de gauche, attaché à la nation mais soucieux de dénoncer les privilèges, Sauvy est plus difficile à classer qu'il n'y paraît, même si sa proximité avec les milieux conservateurs en fin de vie est notoire.

\section{Conclusion}

Au vu de cet ouvrage d'initiation aux questions de population, tout en tenant compte de l'ensemble de son œuvre, que dire de cette volonté farouche de Sauvy «d'éclairer l'action»? D'entrée de jeu, on se rend compte que s'il est désireux avant tout d'éclairer l'action, il n'entend pas être un chercheur enfermé dans sa tour d'ivoire, spéculant à longueur de journée sur des causalités possibles ou probables. L'élaboration de ce qui a été qualifié de «Plan Sauvy» et qui avait pour ambition de jeter «les bases d'une politique économique, à la fois rationnelle et progressiste» (Sauvy, 1960) est une preuve supplémentaire, si cela avait été nécessaire, d'un engagement et même d'une envie d'aller sur un terrain politique, alors même qu'il était encore directeur de l'Ined.

Sauvy conçoit la science de la population comme orientée vers l'action ; le diagnostic doit conduire à la mise en œuvre d'une politique de popu-

30. Fernand Boverat était alors secrétaire général de l’Alliance. 
lation, dans une conception large de celle-ci, c'est-à-dire visant aussi bien la mortalité ou les migrations que la natalité. Si l'activité de prévision est si importante aux yeux de Sauvy, c'est que d'une certaine manière, elle légitime l'action : si le vieillissement annoncé à moyen terme est, par exemple, particulièrement inquiétant, cela signifie alors qu'il faut tout mettre en œuvre pour favoriser une reprise de la natalité.

Telle que Sauvy l'envisage, la démographie est plus une science politique que sociale. Contrairement à Bourgeois-Pichat dans La démographie (1971) mais aussi dans d'autres écrits de ce dernier, Sauvy n'a par exemple aucune préoccupation d'ordre épistémologique. Il évoque régulièrement «la science» mais toujours comme au service du développement économique ou d'un progrès social. Dans tous les autres ouvrages de Sauvy, comme dans les différentes éditions de La population, la démographie apparaît bien comme une science politique. La finalité de la connaissance, c'est l'action.

Dans cet ouvrage d'initiation, dont le succès fut remarquable, Sauvy présente certes les aspects fondamentaux de l'analyse démographique et de la dynamique des populations mais, sur les diverses questions présentées et discutées, il n'hésite pas à prendre parti. Le traitement des informations peut être très normatif, comme le révèle les expressions mêmes utilisées, à travers parfois une violence des mots : à propos de la situation des pays développés, il est question d'«effondrement de la natalité», de «refus de la descendance», de «l'équilibre [...] détruit», de "[l]a masse des retraites [qui] va [...] s'affaisser de son propre poids», des "vieux [qui] écrasent les adultes de leur charge», etc. (LP 1992, p. 112 à 114).

Quelle frontière l'auteur de La population voit-il donc entre activité de recherche et engagement public ? Dans son livre de souvenirs, Sauvy décrit avec précision ce qu'il considère devoir être le positionnement du chercheur. «Il ne s'agit pas, écrit-il, d'abandonner toute idéologie, toute affectivité : le chercheur doit, pendant un moment, se mettre en position de neutralité, d'indifférence, de façon à bien voir, à bien connaître. Une fois informé, une fois enrichi, il est mieux placé pour faire triompher son idéologie ou ses intérêts» (Sauvy, 1981). Tout n'est-il pas dit? 


\section{Références}

ARIES P. (1948), Histoire des populations françaises, Éditions Self, Paris.

ARmengaud A. (1971), "La population, sa mesure, ses mouvements, ses lois», Bibliographie critique, I. Analyses, Population, 26 ème année, 3, p. 591.

BIRABEn J.-N., Dupaquier J. (1981), Les berceaux vides de Marianne. L'avenir de la population française, Éditions du Seuil, Paris.

Bourgeois-Pichat J., Taleb S. A. (1970), «Un taux d'accroissement nul pour les pays en voie de développement en l'an 2000, Rêve ou réalité ?», Population, 5, pp. 957974, http://dx.doi.org/10.2307/1530303.

Bourgeois-Pichat J. (1971), La démographie, Collection «Idées», Paris, Gallimard.

Boverat F. (1942), La résurrection par la natalité, Librairie Hachette, Paris.

Chaunu P., Suffert G. (1976), La peste blanche, Paris Gallimard.

CHesnaIs J.-C. (1990), La démographie, collection «Que-sais-je ?», Paris, Puf.

CLARK C. (1967), Population Growth and Land Use, New York, Saint Martin's Press.

Coale A. J. (1972), The Growth and Structure of Human Populations. A Mathematical Investigation, Princeton University Press, Princeton.

Debre R., Sauvy A. (1946), Des Français pour la France (Le problème de la population), Paris, Gallimard.

Dittgen A. (1992), "La diffusion des connaissances démographiques», Population, 6, p. $1471-1482$.

Dumont G.-F., avec la coll. de Chaunu P., Legrand J., Sauvy A. (1981), La France Ridée, Hachette, Collection Pluriel, Paris.

Feller J. (1975), “'Que sais-je ?' : Une collection qui répond à tout», Communication et langages, 28, pp. 71-75.

GeRARD H., WunsCh G. (1973), Comprendre la démographie, Marabout Université.

GINI C. (1931), Le basi scientifiche della politica della popolazione, Studio Editoriale Moderno, Catania.

GuILLARD A. (1855), Éléments de statistique humaine ou démographie comparée, réédition Ined, 2013.

Hodgson D. (1983), «Demography as Social Science and Policy Science», Population and Development Review, 9 (1), March, pp. 1-34, http://dx.doi.org/10.2307/ 1972893.

KeYfiTz N. (1980), "Population Appearances and Demographic Reality», Population and Development Review, 6 (1), pp. 47-64, http://dx.doi.org/10.2307/1972657.

KeYfitz N. (1985), Applied Mathematical Demography, 2nd Edition, New York and Berlin, Springer-Verlag, http://dx.doi.org/10.1007/978-1-4757-1879-9. 
KeYfiTz N. (1990), "In Memoriam Alfred Sauvy», Population and Development Review, 16 (4), pp. 727-734, http://dx.doi.org/10.2307/1972964.

Klatzmann J. (1975), Nourrir dix milliards d'hommes ?, Paris Puf, réédition en 1983.

LANDRY A. (1929), "Le maximum et l'optimum de population», Scientia, avril, repris dans la réédition Paris, Ined, 1982, pp. 205-215.

LANDRY A. (1934), La Révolution démographique, Paris, Sirey, réédition Paris, Ined, 1982.

LANDRY A. (1945), Traité de démographie, Payot, Paris.

LANDRY A. (1949), Traité de démographie, Payot, Paris.

Ledermann S. (1956), Alcool, alcoolisme et alcoolisation. Données scientifiques de caractère physiologique, économique et social. Tome 1, "Cahiers Travaux et Documents», 29, Ined, Paris, Puf.

Ledermann S. (1964), Alcool, alcoolisme et alcoolisation. Mortalité, morbidité, accidents du travail. Tome 2, "Cahiers Travaux et Documents», 29, Ined, Paris, Puf.

LEVy M.-L. (1990), Alfred Sauvy compagnon du siècle, Paris, La manufacture.

LоткA A. J. (1939), Théorie analytique des associations biologiques, Deuxième partie, Analyse démographique avec application particulière à l'espèce humaine, Paris, Hermann.

Meadows D. H., Meadows D. L., Randers J., Behrens III W. W. (1972). The Limits to Growth, A Potomac Associates Book, New York, traduction française sous le titre Halte à la croissance, Paris, Fayard, 1972.

ReINHARD M. (1949), Histoire de la population mondiale de 1700 à 1948, Édition Domat-Monchrestien, Paris.

Reinhard M., Armengaud A. (1961), Histoire générale de la population mondiale, Montchrestien, Paris.

Reinhard M., Armengaud A., Dupaquier J. (1968), Histoire générale de la population mondiale, Montchrestien, Paris.

Rosental P.-A. (2003), L'intelligence démographique. Sciences et politiques des populations en France (1930-1960), Paris, Odile Jacob.

SAUVy A. (1932), "Calculs démographiques sur la population française jusqu'en 1980", Journal de la Société de Paris, 7, 8, 9, pp. 319-337.

Sauvy A. (1946), «Introduction», Population, 1 (1), pp. 5-8.

SAuvy A. (1956), "Adolphe Landry», Population, 11ème année, 4, pp. 609-620.

Sauvy A. (1958), De Malthus à Mao Tsé-Toung, Paris, Éditions Denoël.

Sauvy A. (1959), La montée des jeunes, Paris Calmann-Lévy.

Sauvy A. (1960), Le plan Sauvy, Paris Calmann-Lévy. 
Sauvy A. (1963), Théorie générale de la population: Économie et croissance, Presses universitaires de France, 2 volumes, Paris.

Sauvy A. (1972), De Paul Reynaud à Charles de Gaulle. Un économiste face aux hommes politiques. 1934-1967, Paris, Casterman.

Sauvy A. (1973), Croissance zéro ?, Paris, Calmann-Lévy.

SAUVy A. (1976), Éléments de démographie, avec la collaboration d'Elisabeth BrowN et d'Alain LefEBVRE, Paris, Thémis, Puf.

Sauvy A. (1978), La tragédie du pouvoir, Paris, Calmann-Lévy.

SAuvy A. (1980), La machine et le chômage, Paris, Dunod.

Sauvy A. (1981), La vie en plus. Souvenirs, Paris, Calmann-Lévy.

SAuvy A. (1987), L'Europe submergée. Sud-Nord dans 30 ans, Paris, Dunod.

SauVy A. (1990), La terre et les hommes, Paris, Economica.

STASSART J. (1965), Les avantages et les inconvénients économiques d'une population stationnaire, Liège-La Haye.

United Nations (2000), Replacement Migration: Is it a Solution to Declining and Ageing Populations?, Population Division, New York.

Veron J. (1998), "Alfred Sauvy aurait cent ans», Population \& Sociétés, 339, www.ined.fr/fichier/t_publication/150/publi_pdf1_pop_et_soc_francais_339.pdf.

Veron J. (1992), "La Théorie générale de la population est-elle toujours une théorie générale de la population ?», Population, 6, pp. 1 411-1 424, http://dx.doi.org/10. 2307/1534076.

Veron J. (1993), Arithmétique de l'Homme. La démographie entre science et politique, Le Seuil, Paris. 


\section{Annexe}

Références figurant en bibliographie dans les différentes éditions de La population $^{31}$

\section{Ouvrages}

ARIEs P. (1948), Histoire des populations françaises, Paris, Éditions Self. [3ème; 15ème]

Bertillon A. (1911), La Dépopulation de la France, Paris, Alcan. [1ère ; 2ème]

BIRABen J.-N., Dupaquier J. (1981), Les berceaux vides de Marianne, Paris, Seuil. [14ème ; 15ème]

BouRgeoIs-PICHAT J. (1971), La démographie, Paris Gallimard NRF, collection «Idées». [11ème ; 13ème]

BoverAT (1942), La Résurrection par la natalité, Paris, Hachette. [1ère]

BOVERAT (1946), Le vieillissement de la population, Paris, Éditions sociales. [2ème ; 5ème]

Chaunu P., Suffert G. (1976), La peste blanche, Paris, Gallimard. [13ème ; 15ème]

Chesnais J. C. (1990), La démographie, "Que sais-je ?», Paris, Puf. [15ème]

Croze M. (1976, 1979, 1982), Tableaux démographiques et sociaux, Paris, Ined. [13ème ; 15ème]

Debre R., Sauvy A. (1946), Des Français pour la France, Paris, Gallimard. [2ème ; 3ème]

Dumont G. F., et al. (1981), La France idée, Paris, Hachette. [14ème ; 15ème]

DUPAQUIER J. (1979), La population française aux 17ème et 18ème siècles, Paris, Puf. [14ème ; 15ème]

EICHER J.-C. (1969), La population, la famille, Paris, Scodel. [10ème ; 11ème]

FreVILLE (1956), L'épouvantail malthusien. [4ème]

Fromont P. (1947), Démographie économique, Paris, Payot. [2ème ; 4ème]

Gerard H., Wunsch G. (1973), Comprendre la démographie, Marabout Université. [12ème ; 15ème]

halbwachs M., Sauvy A., Ulmer H., Bournier G. (1936), «Le point de vue du nombre», Encyclopédie française, Tome VII, Paris, Larousse. [1ère ; 6ème]

31. Entre crochets sont indiquées les éditions encadrantes dans lesquelles chaque référence est citée. Ainsi [3ème ; 15ème] signifie que cette référence est citée dans toutes les éditions de la 3ème à la 15ème incluses. 
HeNRY L. (1960), Leçons d'analyse démographique, Paris, CDU et SEDES. [7ème ; 10ème]

Henry L. (1964), Perspectives démographiques, Paris, Ined. [8ème ; 15ème]

HeNRY L. (1972), Démographie, analyse et modèles, Paris, Larousse. [11ème ; 15ème]

Huber M. (1938-1942), Cours de démographie et de statistique sanitaire, 6 fascicules, Paris, Hermann. [1ère ; 9ème]

Huber M., Bunle H., Boverat F. $(1937,1943,1950)$, La Population de la France, Paris, Hachette, puis Huber M., Bunle H., Boverat F., Febvay M. (1964). [1ère ; 10ème]

Hugon P. (1970), Démographie, Paris, Dalloz. [11ème ; 12ème]

JACQUARD A. (1970), Structures génétiques des populations, Paris, Masson. [12ème]

LAMBerT, PINTo (1944), Problèmes démographiques contemporains, Rio de Janeiro, Atlantica Editors. [3ème]

LANDRY A. (1934), La Révolution démographique, Paris, Sirey. [1ère ; 8ème]

LANDRY A. (1941), La Démographie française, Paris, Puf. [1ère ; 2ème]

LANDRY, et al. (1945, 1949, 1983), Traité de démographie, Paris, Payot. [3ème ; 15ème]

LEGEARD C. (1966), Guide de recherches documentaires en démographie, Paris, Gauthier-Villars. [9ème ; 11ème]

Levasseur E. (1889-1892), La Population française, 3 vol., Paris, Arthur Rousseau. [1ère ; 4ème]

LEVy M.-L. (1982), La population des années 80, Paris, Hatier. [14ème ; 15ème)

LEVy M.-L. (1990), Déchiffrer la démographie, Paris, Syros-Alternatives. [15ème]

Mauco G. (1932), Les Étrangers en France, Paris, Armand Colin. [1ère ; 2ème]

MOHEAU (1778), Recherches et considérations sur la population de la France, réédité à Paris en 1912 chez Geuthner. [1ère ; 4ème]

Mols R. P. (1954), Introduction à la démographie historique des villes d'Europe du 14ème au 18ème siècle, Louvain, Duculot. [5ème ; 10ème]

Paillat P. (1982), Vieillesse et vieillissement, Paris, Puf. [14ème ; 15ème]

Paillat P. (1976), Problèmes démographiques d'aujourd'hui, Paris, Hatier. [14ème ; 15ème]

PнiLIPPot R. (1957), Initiation à une démographie sociale, Paris, Spes. [5ème ; 7ème]

Pressat R. (1961), L'analyse démographique, Paris, Puf. [6ème ; 15ème]

Pressat R. (1967), Pratique de la démographie, Paris, Dunod [9ème ; 13ème]

Pressat R. (1978), Démographie sociale, Paris, Puf [11ème ; 15ème]

Pressat R. (1975), Population et démographie, Paris, Larousse. [13ème] 
Pressat R. (1979), Dictionnaire de démographie, Paris, Puf. [14ème ; 15ème]

Pressat R. (1981), Les méthodes en démographie, Paris, Puf. [14ème ; 15ème]

REINHARD M. (1949), Histoire de la population mondiale de 1700 à 1948, Paris, Domat-Montchrestien; devenu REINHARD M., ARMENGAUd A. (1961), Histoire générale de la population mondiale, Paris, Domat-Montchrestien, puis REINHARD M., ARMENGAUd A., Dupaquier J. (1968), Histoire générale de la population mondiale, Paris, Domat-Montchrestien. [3ème ; 15ème]

SAlleron L., et al. (1946), Malthus a-t-il menti ?, Paris, Albin Michel. [3ème ; 5ème]

Sauvy A. (1943, 1945), Richesse et population, Paris Payot. [1ère ; 3ème]

SAuvy A. (1945), Bien-être et population, Paris, Éditions sociales françaises. [2ème ; 3ème]

SAuvy A. (1952, 1956, 1954, 1959), Théorie générale de la population, I. Économie et croissance, II. Biologie sociale, Paris, Puf, puis SAUVY A. (1952, 1956, 1954, 1959), Théorie générale de la population, I. Économie et croissance, II. La vie des populations, (1963, 1966), Paris, Puf. [4ème ; 15ème]

Sauvy A. (1958), De Malthus à Mao-Tsé-Toung, Paris, Denoël. [5ème ; 7ème]

Sauvy A. (1961), Les limites de la vie humaine, Paris, Hachette. [6ème ; 12ème]

SAuvy A. (1962, 1965, 1967), La prévention des naissances, Paris, Puf. [7ème ; 11ème]

Sauvy A. (1963, 1966), Malthus et les deux Marx, Paris, Denoël. [7ème ; 12ème]

Sauvy A. (1973), Croissance zéro, Paris, Calmann-Lévy. [12ème ; 13ème]

Sauvy A. (1975), La fin des riches, Paris, Calmann-Lévy. [12ème ; 15ème]

SAuvy A. (1977), Coût et valeur de la vie humaine, Paris, Hermann. [13ème ; 15ème]

Sauvy A. (1977), Éléments de démographie, Paris, Puf. [13ème ; 15ème]

Sauvy A. (1982), Mondes en marche, Paris, Calmann-Lévy. [14ème ; 15ème]

STASSART J. (1957), Malthus et la population, Liège, Faculté de Droit. [5ème ; 8ème]

STASSART J. (1965), Les avantages et les inconvénients économiques d'une population stationnaire, Liège, Faculté de Droit, La Haye Martinus Nijhoff. [8ème ; 12ème]

Union internationale pour l'étude scientifique de la population (1981), Dictionnaire démographique multilingue, L. HENRY (ed), Ordina Éditions, Liège.

WLocevskı S. (1938), L'installation des Italiens en France, Paris, Alcan. [1ère]

Recueils statistiques, publications périodiques et autres

Annuaire statistique de la France, Imprimerie nationale et Berger-Levrault. [1ère ; 7ème]

Annuaire statistique de la Société des Nations, Genève. [2ème] 
Annuaire démographique et Bulletin statistique des Nations Unies, Nations unies, New York. [4ème ; 15ème]

Statistique annuelle du mouvement de la population, Berger-Levrault et Imprimerie nationale. [1ère ; 7ème]

Résultats statistiques du recensement de la population, Berger-Levrault et Imprimerie nationale. [1ère ; 7ème]

Reproduction nette en Europe depuis les origines de l'état civil (1941), BergerLevrault et Imprimerie nationale. [1ère ; 2ème]

Évolution de la mortalité en Europe depuis les origines de l'état civil (1941), BergerLevrault et Imprimerie nationale. [1ère ; 2ème]

Les Naturalisations en France 1870-1940 (1942), Berger-Levrault et Imprimerie nationale. [1ère ; 2ème]

Mouvements migratoires entre la France et l'étranger (1943), Berger-Levrault et Imprimerie nationale. [1ère ; 2ème]

Dictionnaire démographique multilingue, Nations unies, New York. [5ème ; 15ème]

Documentation Française (1978), "La France et sa population», Cahiers français, 184. [13ème ; 15ème]

Statistique annuelle du mouvement de la population et Résultats statistiques du recensement de la population, Imprimerie nationale, puis Paris, Insee. [2ème ; 15ème]

Revue Population. [2ème ; 15ème]

Cahiers de l'Ined. [2ème ; 15ème]

Manuel élémentaire de démographie française (1965), Paris, Alliance nationale «pour la vitalité française», etc. [6ème ; 11ème]

Publications de l'Alliance nationale contre la dépopulation, notamment la Revue de I'Alliance nationale. [1ère ; 2ème] 